2

3

4

5

6

7

8

9

10

11

12

13

14

15

16

17

18

19

20

21

22

$23 *$ Corresponding author: email: heyden@cec.sc.edu

8

9

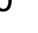

\title{
Theoretical Investigation of Solvent Effects on the Hydrodeoxygenation of Propionic Acid over a Ni (111) Catalyst Model
}

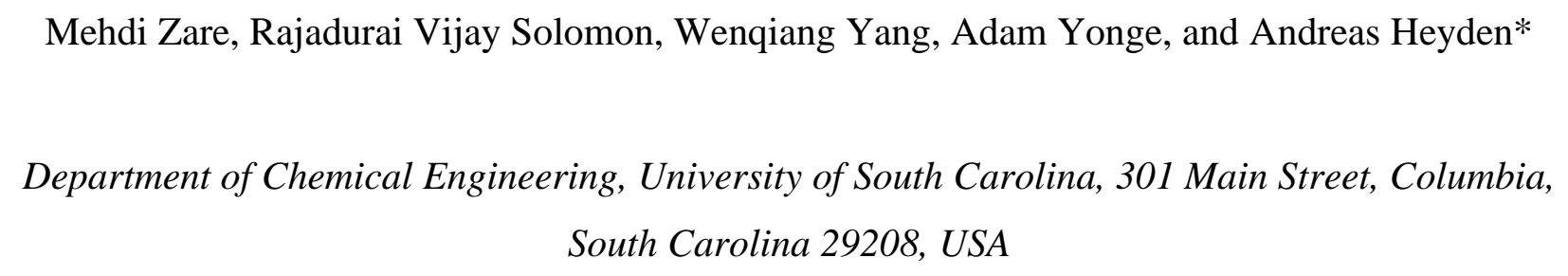

\begin{abstract}
Department of Chemical Engineering, University of South Carolina, 301 Main Street, Columbia,
\end{abstract}


2 Table S1. Solvent effect on the stability of various adsorbed species in the propionic acid conversion to ethane and 3 ethylene over a $\mathrm{Ni}$ (111) catalyst surface model at two different temperatures of $298 \mathrm{~K}$ and $473 \mathrm{~K}$ based on two implicit 4 solvation schemes: iSMS and VASPsol. $\Delta \Delta \mathrm{G}_{\mathrm{rxn}}$ indicates the difference in the adsorption free energy of the 5 corresponding intermediates in the presence and absence of water. Asterisk $(*)$ represents a surface adsorption site 6 and multiple asterisks are indicative of the number of occupied active sites.

\begin{tabular}{|c|c|c|c|c|}
\hline \multirow{3}{*}{ Adsorbed species } & \multicolumn{4}{|c|}{$\Delta \Delta G_{r \times n}, e V$} \\
\hline & \multicolumn{2}{|c|}{$\mathrm{T}=298 \mathrm{~K}$} & \multicolumn{2}{|c|}{$\mathrm{T}=473 \mathrm{~K}$} \\
\hline & iSMS & VASPsol & iSMS & VASPsol \\
\hline $\mathrm{CH}_{2} \mathrm{C}^{\star \star \star}$ & -0.04 & 0.02 & -0.08 & 0.05 \\
\hline $\mathrm{CH}_{2} \mathrm{CH}^{\star \star \star}$ & 0.02 & -0.08 & -0.01 & -0.07 \\
\hline $\mathrm{CH}_{2} \mathrm{CH}_{2}{ }^{\star \star}$ & 0.06 & -0.08 & 0.10 & -0.08 \\
\hline $\mathrm{CH}_{2} \mathrm{CHCO}^{\star \star \star \star}$ & -0.05 & -0.07 & -0.09 & -0.05 \\
\hline $\mathrm{CH}_{2} \mathrm{CHCOOH}^{\star \star \star *}$ & -0.10 & -0.34 & -0.11 & -0.29 \\
\hline $\mathrm{CH}_{3} \mathrm{C}^{\star \star \star}$ & 0.02 & -0.04 & -0.01 & -0.03 \\
\hline $\mathrm{CH}_{3} \mathrm{CCO}^{\star \star \star \star \star}$ & -0.09 & -0.07 & -0.12 & -0.06 \\
\hline $\mathrm{CH}_{3} \mathrm{CCOO}$ *** & -0.63 & -0.29 & -0.55 & -0.26 \\
\hline $\mathrm{CH}_{3} \mathrm{CCOOH}^{\star \star *}$ & -0.12 & -0.28 & -0.12 & -0.26 \\
\hline $\mathrm{CH}_{3} \mathrm{CH}^{\star \star \star}$ & 0.03 & -0.05 & 0.00 & -0.03 \\
\hline $\mathrm{CH}_{3} \mathrm{CH}_{2}{ }^{\star \star}$ & 0.03 & -0.06 & 0.01 & -0.05 \\
\hline $\mathrm{CH}_{3} \mathrm{CH}_{2} \mathrm{CO}^{\star \star \star}$ & -0.02 & -0.11 & -0.05 & -0.12 \\
\hline $\mathrm{CH}_{3} \mathrm{CH}_{2} \mathrm{COO}^{* *}$ & -0.05 & -0.11 & -0.06 & -0.10 \\
\hline $\mathrm{CH}_{3} \mathrm{CH}_{2} \mathrm{COOH}^{*}$ & -0.07 & -0.44 & -0.03 & -0.40 \\
\hline $\mathrm{CH}_{3} \mathrm{CH}_{3}{ }^{*}$ & 0.05 & -0.04 & 0.02 & -0.06 \\
\hline $\mathrm{CH}_{3} \mathrm{CHCO}^{\star *}$ & -0.06 & -0.11 & -0.09 & -0.11 \\
\hline $\mathrm{CH}_{3} \mathrm{CHCOO} * \star \star$ & -0.34 & -0.12 & -0.31 & -0.10 \\
\hline $\mathrm{CH}_{3} \mathrm{CHCOOH}^{\star \star}$ & -0.10 & -0.30 & -0.10 & -0.28 \\
\hline $\mathrm{CHCH}^{\star \star \star \star}$ & 0.02 & -0.06 & 0.05 & -0.05 \\
\hline $\mathrm{CHCHCO}{ }^{\star \star \star \star}$ & -0.11 & -0.12 & -0.15 & -0.10 \\
\hline $\mathrm{CHCHCOOH}{ }^{* * * *}$ & -0.13 & -0.31 & -0.13 & -0.26 \\
\hline $\mathrm{CO}^{\star * *}$ & -0.15 & -0.08 & -0.25 & -0.08 \\
\hline $\mathrm{CO}_{2}{ }^{*}$ & 0.05 & -0.10 & -0.04 & -0.07 \\
\hline $\mathrm{COOH}^{\star *}$ & -0.18 & -0.25 & -0.16 & -0.21 \\
\hline $\mathrm{H}^{*}$ & -0.02 & 0.00 & -0.01 & 0.00 \\
\hline $\mathrm{H}_{2} \mathrm{O}^{*}$ & -0.04 & -0.31 & 0.02 & -0.25 \\
\hline $\mathrm{OH}^{*}$ & -0.02 & -0.21 & 0.00 & -0.18 \\
\hline
\end{tabular}


2 Table S2. Solvent effect on the stability of transition states in the propionic acid conversion to ethane and ethylene 3 over a Ni (111) catalyst surface model at two different temperatures of $298 \mathrm{~K}$ and $473 \mathrm{~K}$ based on two implicit 4 solvation schemes: iSMS and VASPsol. Solvent effect on TS indicates the difference in the adsorption free energy of 5 the corresponding transition state in the presence and absence of water.

\begin{tabular}{|c|c|c|c|c|c|}
\hline \multirow{3}{*}{$\#$} & \multirow{3}{*}{ Reaction } & \multicolumn{4}{|c|}{ Solvent effect on TS, eV } \\
\hline & & \multicolumn{2}{|c|}{$T=298 \mathrm{~K}$} & \multicolumn{2}{|c|}{$\mathrm{T}=473 \mathrm{~K}$} \\
\hline & & iSMS & VASPsol & iSMS & VASPsol \\
\hline 1 & $\mathrm{CH}_{3} \mathrm{CH}_{2} \mathrm{COOH}^{*}+3^{*} \rightarrow \mathrm{CH}_{3} \mathrm{CH}_{2} \mathrm{CO}^{\star \star *}+\mathrm{OH}^{*}$ & -0.03 & -0.16 & -0.05 & -0.11 \\
\hline 2 & $\mathrm{CH}_{3} \mathrm{CH}_{2} \mathrm{COOH}^{\star}+2^{\star} \rightarrow \mathrm{CH}_{3} \mathrm{CHCOOH}^{\star \star}+\mathrm{H}^{\star}$ & -0.10 & -0.39 & -0.09 & -0.37 \\
\hline 3 & $\mathrm{CH}_{3} \mathrm{CH}_{2} \mathrm{CO}^{\star * *}+2^{*} \rightarrow \mathrm{CH}_{3} \mathrm{CH}_{2}{ }^{* *}+\mathrm{CO}^{\star * *}$ & -0.04 & -0.10 & -0.07 & -0.10 \\
\hline 4 & $\mathrm{CH}_{3} \mathrm{CH}_{2} \mathrm{CO}^{\star \star *} \rightarrow \mathrm{CH}_{3} \mathrm{CHCO}^{\star *}+\mathrm{H}^{\star}$ & -0.05 & -0.10 & -0.08 & -0.07 \\
\hline 5 & $\mathrm{CH}_{3} \mathrm{CHCOOH}^{\star *}+* \rightarrow \mathrm{CH}_{3} \mathrm{CHCO}^{* \star}+\mathrm{OH}^{*}$ & -0.12 & -0.17 & -0.14 & -0.15 \\
\hline 6 & $\mathrm{CH}_{3} \mathrm{CHCOOH}^{* *}+3^{*} \rightarrow \mathrm{CH}_{2} \mathrm{CHCOOH}^{\star * * *}+\mathrm{H}^{*}$ & -0.11 & -0.36 & -0.10 & -0.32 \\
\hline 7 & $\mathrm{CH}_{3} \mathrm{CHCOOH}^{\star \star}+2^{*} \rightarrow \mathrm{CH}_{3} \mathrm{CCOOH}^{\star \star \star}+\mathrm{H}^{*}$ & -0.10 & -0.30 & -0.10 & -0.25 \\
\hline 8 & $\mathrm{CH}_{3} \mathrm{CHCO}^{* *}+4^{*} \rightarrow \mathrm{CH}_{3} \mathrm{CH}^{* * *}+\mathrm{CO}^{* * *}$ & -0.06 & -0.11 & -0.10 & -0.11 \\
\hline 9 & $\mathrm{CH}_{3} \mathrm{CHCO}^{* *}+3^{*} \rightarrow \mathrm{CH}_{3} \mathrm{CCO}^{\star * \star *}+\mathrm{H}^{*}$ & -0.13 & -0.07 & -0.15 & -0.04 \\
\hline 10 & $\mathrm{CH}_{3} \mathrm{CHCO}^{* *}+3^{*} \rightarrow \mathrm{CH}_{2} \mathrm{CHCO}^{\star * * *}+\mathrm{H}^{*}$ & -0.05 & -0.13 & -0.09 & -0.10 \\
\hline 11 & $\mathrm{CH}_{2} \mathrm{CHCOOH}^{\star \star \star *}+* \rightarrow \mathrm{CH}_{2} \mathrm{CHCO}^{\star \star \star *}+\mathrm{OH}^{*}$ & -0.08 & -0.19 & -0.11 & -0.16 \\
\hline 12 & $\mathrm{CH}_{2} \mathrm{CHCOOH}^{\star \star \star *}+{ }^{*} \rightarrow \mathrm{CHCHCOOH}^{\star \star \star *}+\mathrm{H}^{*}$ & -0.10 & -0.34 & -0.10 & -0.30 \\
\hline 13 & $\mathrm{CH}_{3} \mathrm{CCOOH}^{\star \star \star}+2^{\star} \rightarrow \mathrm{CH}_{3} \mathrm{CCO}^{\star \star \star \star}+\mathrm{OH}^{*}$ & -0.18 & -0.19 & -0.20 & -0.17 \\
\hline 14 & $\mathrm{CH}_{3} \mathrm{CCO}^{\star \star \star *}+2^{*} \rightarrow \mathrm{CH}_{3} \mathrm{C}^{\star * \star}+\mathrm{CO}^{\star \star *}$ & -0.17 & -0.09 & -0.20 & -0.07 \\
\hline 15 & $\mathrm{CH}_{2} \mathrm{CHCO}^{\star \star \star \star}+2^{\star} \rightarrow \mathrm{CH}_{2} \mathrm{CH}^{\star \star \star}+\mathrm{CO}^{\star \star \star}$ & -0.05 & -0.12 & -0.09 & -0.10 \\
\hline 16 & $\mathrm{CH}_{2} \mathrm{CHCO}^{\star * * *}+{ }^{*} \rightarrow \mathrm{CHCHCO}{ }^{* * * *}+\mathrm{H}^{*}$ & -0.05 & -0.11 & -0.09 & -0.10 \\
\hline 17 & $\mathrm{CHCHCOOH}^{* \star * *}+* \rightarrow \mathrm{CHCHCO}{ }^{* \star * *}+\mathrm{OH}^{*}$ & -0.24 & -0.17 & -0.26 & -0.14 \\
\hline 18 & $\mathrm{CHCHCO}^{\star \star \star *}+3^{\star} \rightarrow \mathrm{CHCH}^{\star \star \star *}+\mathrm{CO}^{\star \star *}$ & -0.13 & -0.12 & -0.17 & -0.11 \\
\hline 19 & $\mathrm{CH}_{2} \mathrm{CH}^{\star \star \star}+2^{\star} \rightarrow \mathrm{CHCH}^{\star \star \star *}+\mathrm{H}^{\star}$ & 0.05 & -0.08 & 0.01 & -0.07 \\
\hline 20 & $\mathrm{CH}_{2} \mathrm{CH}_{2}{ }^{\star \star}+2^{\star} \rightarrow \mathrm{CH}_{2} \mathrm{CH}^{\star \star \star}+\mathrm{H}^{\star}$ & 0.07 & -0.09 & 0.04 & -0.09 \\
\hline 21 & $\mathrm{CH}_{2} \mathrm{CH}^{\star \star *}+{ }^{*} \rightarrow \mathrm{CH}_{2} \mathrm{C}^{\star \star *}+\mathrm{H}^{*}$ & 0.01 & -0.05 & -0.03 & -0.05 \\
\hline 22 & $\mathrm{CH}_{3} \mathrm{C}^{\star \star \star}+{ }^{*} \rightarrow \mathrm{CH}_{2} \mathrm{C}^{\star \star \star}+\mathrm{H}^{\star}$ & 0.01 & -0.08 & -0.02 & -0.07 \\
\hline 23 & $\mathrm{CH}_{3} \mathrm{CH}^{\star \star \star}+{ }^{*} \rightarrow \mathrm{CH}_{2} \mathrm{CH}^{\star \star \star}+\mathrm{H}^{\star}$ & 0.00 & -0.10 & -0.03 & -0.09 \\
\hline 24 & $\mathrm{CH}_{3} \mathrm{CH}^{\star \star \star}+* \rightarrow \mathrm{CH}_{3} \mathrm{C}^{\star \star \star}+\mathrm{H}^{\star}$ & 0.04 & -0.05 & 0.01 & -0.04 \\
\hline 25 & $\mathrm{CH}_{3} \mathrm{CH}_{2}{ }^{\star \star}+2^{\star} \rightarrow \mathrm{CH}_{3} \mathrm{CH}^{\star \star \star}+\mathrm{H}^{\star}$ & 0.05 & -0.05 & 0.02 & -0.02 \\
\hline 26 & $\mathrm{CH}_{3} \mathrm{CH}_{3}{ }^{*}+2^{*} \rightarrow \mathrm{CH}_{3} \mathrm{CH}_{2}{ }^{* *}+\mathrm{H}^{*}$ & 0.04 & -0.08 & 0.02 & -0.08 \\
\hline 27 & $\mathrm{CH}_{3} \mathrm{CH}_{2}{ }^{* *}+{ }^{*} \rightarrow \mathrm{CH}_{2} \mathrm{CH}_{2}{ }^{* *}+\mathrm{H}^{*}$ & 0.03 & -0.13 & 0.00 & -0.11 \\
\hline 28 & $\mathrm{CH}_{3} \mathrm{CH}_{2} \mathrm{COOH}^{*}+2^{\star} \rightarrow \mathrm{CH}_{3} \mathrm{CH}_{2} \mathrm{COO}^{\star *}+\mathrm{H}^{*}$ & -0.11 & -0.15 & -0.12 & -0.16 \\
\hline 29 & $\mathrm{CH}_{3} \mathrm{CH}_{2} \mathrm{COO}^{\star *}+{ }^{*} \rightarrow \mathrm{CH}_{3} \mathrm{CH}_{2}{ }^{\star \star}+\mathrm{CO}_{2}{ }^{*}$ & -0.06 & -0.12 & -0.10 & -0.09 \\
\hline 30 & $\mathrm{CH}_{3} \mathrm{CH} 2 \mathrm{COO}^{* *}+2^{*} \rightarrow \mathrm{CH}_{3} \mathrm{CHCOO}^{* * *}+\mathrm{H}^{*}$ & -0.24 & -0.12 & -0.23 & -0.11 \\
\hline 31 & $\mathrm{CH}_{3} \mathrm{CHCOOH}^{\star \star}+2^{*} \rightarrow \mathrm{CH}_{3} \mathrm{CHCOO}^{\star \star \star}+\mathrm{H}^{*}$ & -0.24 & -0.10 & -0.25 & -0.09 \\
\hline 32 & $\mathrm{CH}_{3} \mathrm{CHCOOH}^{\star \star}+3^{*} \rightarrow \mathrm{CH}_{3} \mathrm{CH}^{\star \star \star}+\mathrm{COOH}^{\star \star}$ & -0.12 & -0.29 & -0.12 & -0.23 \\
\hline 33 & $\mathrm{CH}_{3} \mathrm{CHCOO}^{\star * \star}+{ }^{*} \rightarrow \mathrm{CH}_{3} \mathrm{CH}^{\star * *}+\mathrm{CO}_{2}{ }^{*}$ & -0.18 & -0.12 & -0.19 & -0.10 \\
\hline 34 & $\mathrm{CH}_{3} \mathrm{CHCOO}^{* \star *}+* \rightarrow \mathrm{CH}_{3} \mathrm{CCOO}^{* * *}+\mathrm{H}^{*}$ & -0.20 & -0.09 & -0.20 & -0.08 \\
\hline 35 & $\mathrm{CH}_{3} \mathrm{CCOOH}^{\star \star *}+* \rightarrow \mathrm{CH}_{3} \mathrm{CCOO}^{\star * *}+\mathrm{H}^{*}$ & -0.32 & -0.16 & -0.30 & -0.15 \\
\hline 36 & $\mathrm{CH}_{3} \mathrm{CCOOH}^{\star \star \star}+2^{\star} \rightarrow \mathrm{CH}_{3} \mathrm{C}^{\star \star \star}+\mathrm{COOH}^{\star \star}$ & -0.16 & -0.30 & -0.17 & -0.25 \\
\hline 37 & $\mathrm{CH}_{2} \mathrm{CHCOOH}^{\star \star \star \star}+* \rightarrow \mathrm{CH}_{2} \mathrm{CH}^{\star \star *}+\mathrm{COOH}^{\star \star}$ & -0.11 & -0.32 & -0.11 & -0.26 \\
\hline 38 & $\mathrm{CH}_{3} \mathrm{CCOO}^{\star \star *}+{ }^{*} \rightarrow \mathrm{CH}_{3} \mathrm{C}^{\star * *}+\mathrm{CO}_{2}{ }^{*}$ & -0.34 & -0.16 & -0.35 & -0.15 \\
\hline 39 & $\mathrm{COOH}^{\star *} \rightarrow \mathrm{CO}_{2}^{*}+\mathrm{H}^{\star}$ & -0.34 & -0.17 & -0.34 & -0.15 \\
\hline 40 & $\mathrm{COOH}^{\star *}+2^{*} \rightarrow \mathrm{CO}^{\star * *}+\mathrm{OH}^{*}$ & -0.12 & -0.25 & -0.12 & -0.23 \\
\hline 41 & $\mathrm{H}_{2} \mathrm{O}^{*}+{ }^{*} \rightarrow \mathrm{OH}^{*}+\mathrm{H}^{*}$ & -0.04 & -0.53 & -0.03 & -0.58 \\
\hline
\end{tabular}


1 Table S3. Solvent effect on the reaction free energies of all elementary surface reactions of the propionic acid 2 conversion to ethane and ethylene over a $\mathrm{Ni}$ (111) catalyst surface model at two different temperature of $298 \mathrm{~K}$ and $3473 \mathrm{~K}$ based on two different implicit solvation schemes: iSMS and VASPsol. $\Delta \Delta \mathrm{G}_{\mathrm{rxn}}$ indicates the reaction free 4 energy difference between corresponding reaction in liquid water and in a gas phase.

\begin{tabular}{|c|c|c|c|c|c|}
\hline \multirow{3}{*}{ \# } & \multirow{3}{*}{ Reaction } & \multicolumn{4}{|c|}{$\Delta \Delta \mathbf{G}_{\mathrm{rxn}}$} \\
\hline & & \multicolumn{2}{|c|}{$\mathrm{T}=298 \mathrm{~K}$} & \multicolumn{2}{|c|}{$\mathrm{T}=473 \mathrm{~K}$} \\
\hline & & iSMS & VASPsol & iSMS & VASPsol \\
\hline 1 & $\mathrm{CH}_{3} \mathrm{CH}_{2} \mathrm{COOH}^{*}+3^{*} \rightarrow \mathrm{CH}_{3} \mathrm{CH}_{2} \mathrm{CO}^{* * *}+\mathrm{OH}^{*}$ & 0.03 & 0.13 & 0.00 & 0.10 \\
\hline 2 & $\mathrm{CH}_{3} \mathrm{CH}_{2} \mathrm{COOH}^{\star}+2^{*} \rightarrow \mathrm{CH}_{3} \mathrm{CHCOOH}^{\star *}+\mathrm{H}^{\star}$ & -0.04 & 0.14 & -0.07 & 0.12 \\
\hline 3 & $\mathrm{CH}_{3} \mathrm{CH}_{2} \mathrm{CO}^{\star \star *}+2^{\star} \rightarrow \mathrm{CH}_{3} \mathrm{CH}_{2}{ }^{\star *}+\mathrm{CO}^{\star \star *}$ & -0.10 & -0.04 & -0.14 & -0.01 \\
\hline 4 & $\mathrm{CH}_{3} \mathrm{CH}_{2} \mathrm{CO}^{\star \star *} \rightarrow \mathrm{CH}_{3} \mathrm{CHCO}^{\star *}+\mathrm{H}^{*}$ & -0.05 & 0.00 & -0.06 & 0.01 \\
\hline 5 & $\mathrm{CH}_{3} \mathrm{CHCOOH}^{\star \star}+{ }^{*} \rightarrow \mathrm{CH}_{3} \mathrm{CHCO}^{* \star}+\mathrm{OH}^{*}$ & 0.02 & -0.01 & 0.01 & -0.01 \\
\hline 6 & $\mathrm{CH}_{3} \mathrm{CHCOOH}^{\star \star}+3^{\star} \rightarrow \mathrm{CH}_{2} \mathrm{CHCOOH}^{\star \star \star \star}+\mathrm{H}^{\star}$ & -0.02 & -0.03 & -0.02 & -0.01 \\
\hline 7 & $\mathrm{CH}_{3} \mathrm{CHCOOH}^{\star \star}+2^{*} \rightarrow \mathrm{CH}_{3} \mathrm{CCOOH}^{\star \star \star}+\mathrm{H}^{*}$ & -0.03 & 0.02 & -0.03 & 0.02 \\
\hline 8 & $\mathrm{CH}_{3} \mathrm{CHCO}^{\star \star}+4^{\star} \rightarrow \mathrm{CH}_{3} \mathrm{CH}^{\star \star \star}+\mathrm{CO}^{\star \star \star}$ & -0.07 & -0.03 & -0.10 & 0.00 \\
\hline 9 & $\mathrm{CH}_{3} \mathrm{CHCO}^{\star \star}+3^{\star} \rightarrow \mathrm{CH}_{3} \mathrm{CCO}^{\star \star \star *}+\mathrm{H}^{*}$ & -0.05 & 0.04 & -0.05 & 0.06 \\
\hline 10 & $\mathrm{CH}_{3} \mathrm{CHCO}^{\star \star}+3^{\star} \rightarrow \mathrm{CH}_{2} \mathrm{CHCO}^{\star \star \star \star}+\mathrm{H}^{*}$ & -0.01 & 0.04 & -0.01 & 0.06 \\
\hline 11 & $\mathrm{CH}_{2} \mathrm{CHCOOH}^{\star \star \star *}+{ }^{*} \rightarrow \mathrm{CH}_{2} \mathrm{CHCO}^{\star \star \star *}+\mathrm{OH}^{*}$ & 0.03 & 0.06 & 0.02 & 0.06 \\
\hline 12 & $\mathrm{CH}_{2} \mathrm{CHCOOH}^{\star \star \star \star}+* \rightarrow \mathrm{CHCHCOOH}^{\star \star \star \star}+\mathrm{H}^{*}$ & -0.05 & 0.04 & -0.05 & 0.03 \\
\hline 13 & $\mathrm{CH}_{3} \mathrm{CCOOH}^{\star \star \star}+2^{\star} \rightarrow \mathrm{CH}_{3} \mathrm{CCO}^{\star \star \star *}+\mathrm{OH}^{\star}$ & 0.01 & 0.01 & -0.01 & 0.02 \\
\hline 14 & $\mathrm{CH}_{3} \mathrm{CCO}^{\star \star \star \star}+2^{*} \rightarrow \mathrm{CH}_{3} \mathrm{C}^{\star \star *}+\mathrm{CO}^{\star \star \star}$ & -0.04 & -0.06 & -0.08 & -0.05 \\
\hline 15 & $\mathrm{CH}_{2} \mathrm{CHCO}^{\star \star \star \star}+2^{\star} \rightarrow \mathrm{CH}_{2} \mathrm{CH}^{\star \star \star}+\mathrm{CO}^{\star \star \star}$ & -0.07 & -0.09 & -0.11 & -0.10 \\
\hline 16 & $\mathrm{CH}_{2} \mathrm{CHCO}^{* * * *}+{ }^{*} \rightarrow \mathrm{CHCHCO}^{\star * * *}+\mathrm{H}^{*}$ & -0.08 & -0.04 & -0.08 & -0.05 \\
\hline 17 & $\mathrm{CHCHCOOH}^{* * * *}+{ }^{*} \rightarrow \mathrm{CHCHCO}{ }^{* * * *}+\mathrm{OH}^{*}$ & 0.00 & -0.02 & -0.01 & -0.03 \\
\hline 18 & $\mathrm{CHCHCO}^{\star \star \star *}+3^{\star} \rightarrow \mathrm{CHCH}^{\star \star \star *}+\mathrm{CO}^{\star \star \star}$ & -0.02 & -0.02 & -0.06 & -0.03 \\
\hline 19 & $\mathrm{CH}_{2} \mathrm{CH}^{\star \star \star}+2^{\star} \rightarrow \mathrm{CHCH}^{\star \star \star *}+\mathrm{H}^{*}$ & -0.02 & 0.02 & -0.03 & 0.02 \\
\hline 20 & $\mathrm{CH}_{2} \mathrm{CH}_{2}{ }^{\star \star}+2^{\star} \rightarrow \mathrm{CH}_{2} \mathrm{CH}^{\star \star \star}+\mathrm{H}^{\star}$ & -0.05 & 0.00 & -0.06 & 0.01 \\
\hline 21 & $\mathrm{CH}_{2} \mathrm{CH}^{\star \star \star}+* \rightarrow \mathrm{CH}_{2} \mathrm{C}^{\star \star \star}+\mathrm{H}^{\star}$ & -0.08 & 0.10 & -0.09 & 0.13 \\
\hline 22 & $\mathrm{CH}_{3} \mathrm{C}^{\star \star *}+{ }^{*} \rightarrow \mathrm{CH}_{2} \mathrm{C}^{\star \star *}+\mathrm{H}^{\star}$ & -0.07 & 0.06 & -0.08 & 0.08 \\
\hline 23 & $\mathrm{CH}_{3} \mathrm{CH}^{\star \star \star}+{ }^{*} \rightarrow \mathrm{CH}_{2} \mathrm{CH}^{\star \star \star}+\mathrm{H}^{*}$ & -0.02 & -0.02 & -0.03 & -0.04 \\
\hline 24 & $\mathrm{CH}_{3} \mathrm{CH}^{\star \star \star}+* \rightarrow \mathrm{CH}_{3} \mathrm{C}^{\star \star \star}+\mathrm{H}^{*}$ & -0.02 & 0.01 & -0.03 & 0.01 \\
\hline 25 & $\mathrm{CH}_{3} \mathrm{CH}_{2}{ }^{* *}+2^{\star} \rightarrow \mathrm{CH}_{3} \mathrm{CH}^{\star \star *}+\mathrm{H}^{*}$ & -0.02 & 0.02 & -0.03 & 0.02 \\
\hline 26 & $\mathrm{CH}_{3} \mathrm{CH}_{3}{ }^{*}+2^{\star} \rightarrow \mathrm{CH}_{3} \mathrm{CH}_{2}{ }^{* *}+\mathrm{H}^{*}$ & -0.04 & -0.02 & -0.05 & 0.02 \\
\hline 27 & $\mathrm{CH}_{3} \mathrm{CH}_{2}{ }^{* *}+{ }^{*} \rightarrow \mathrm{CH}_{2} \mathrm{CH}_{2}{ }^{* *}+\mathrm{H}^{*}$ & 0.01 & -0.01 & 0.01 & -0.02 \\
\hline 28 & $\mathrm{CH}_{3} \mathrm{CH}_{2} \mathrm{COOH}^{*}+2^{*} \rightarrow \mathrm{CH}_{3} \mathrm{CH}_{2} \mathrm{COO}^{* *}+\mathrm{H}^{*}$ & 0.01 & 0.33 & -0.03 & 0.30 \\
\hline 29 & $\mathrm{CH}_{3} \mathrm{CH}_{2} \mathrm{COO}^{\star *}+{ }^{*} \rightarrow \mathrm{CH}_{3} \mathrm{CH}_{2}{ }^{\star *}+\mathrm{CO}_{2}{ }^{*}$ & 0.13 & -0.05 & 0.06 & -0.02 \\
\hline 30 & $\mathrm{CH}_{3} \mathrm{CH}_{2} \mathrm{COO}^{\star \star}+2^{\star} \rightarrow \mathrm{CH}_{3} \mathrm{CHCOO}^{\star \star *}+\mathrm{H}^{\star}$ & -0.31 & -0.01 & -0.27 & 0.00 \\
\hline 31 & $\mathrm{CH}_{3} \mathrm{CHCOOH}^{\star \star}+2^{\star} \rightarrow \mathrm{CH}_{3} \mathrm{CHCOO}^{\star \star *}+\mathrm{H}^{*}$ & -0.26 & 0.19 & -0.23 & 0.18 \\
\hline 32 & $\mathrm{CH}_{3} \mathrm{CHCOOH}^{\star \star}+3^{\star} \rightarrow \mathrm{CH}_{3} \mathrm{CH}^{\star \star \star}+\mathrm{COOH}^{\star \star}$ & -0.05 & 0.01 & -0.06 & 0.04 \\
\hline 33 & $\mathrm{CH}_{3} \mathrm{CHCOO}^{\star \star *}+{ }^{*} \rightarrow \mathrm{CH}_{3} \mathrm{CH}^{\star \star *}+\mathrm{CO}_{2}^{*}$ & 0.42 & -0.03 & 0.31 & 0.00 \\
\hline 34 & $\mathrm{CH}_{3} \mathrm{CHCOO}^{\star * *}+{ }^{*} \rightarrow \mathrm{CH}_{3} \mathrm{CCOO}^{\star * *}+\mathrm{H}^{*}$ & -0.30 & -0.18 & -0.26 & -0.14 \\
\hline 35 & $\mathrm{CH}_{3} \mathrm{CCOOH}^{\star \star *}+{ }^{*} \rightarrow \mathrm{CH}_{3} \mathrm{CCOO}^{\star * *}+\mathrm{H}^{*}$ & -0.52 & 0.00 & -0.45 & 0.00 \\
\hline 36 & $\mathrm{CH}_{3} \mathrm{CCOOH}^{* \star *}+2^{*} \rightarrow \mathrm{CH}_{3} \mathrm{C}^{* * *}+\mathrm{COOH}^{\star *}$ & -0.04 & -0.01 & -0.06 & 0.02 \\
\hline 37 & $\mathrm{CH}_{2} \mathrm{CHCOOH}^{\star \star * *}+{ }^{*} \rightarrow \mathrm{CH}_{2} \mathrm{CH}^{* * *}+\mathrm{COOH}^{* *}$ & -0.05 & 0.02 & -0.07 & 0.01 \\
\hline 38 & $\mathrm{CH}_{3} \mathrm{CCOO}^{\star \star \star}+{ }^{*} \rightarrow \mathrm{CH}_{3} \mathrm{C}^{\star \star *}+\mathrm{CO}_{2}^{*}$ & 0.70 & 0.15 & 0.54 & 0.16 \\
\hline 39 & $\mathrm{COOH}^{\star *} \rightarrow \mathrm{CO}_{2}^{*}+\mathrm{H}^{*}$ & 0.22 & 0.15 & 0.15 & 0.14 \\
\hline 40 & $\mathrm{COOH}^{* *}+2^{*} \rightarrow \mathrm{CO}^{* * *}+\mathrm{OH}^{*}$ & 0.01 & -0.04 & -0.03 & -0.06 \\
\hline 41 & $\mathrm{H}_{2} \mathrm{O}^{*}+{ }^{*} \rightarrow \mathrm{OH}^{*}+\mathrm{H}^{*}$ & 0.00 & 0.11 & 0.00 & 0.07 \\
\hline
\end{tabular}


Table S4. Solvent effect on the activation free energies of all elementary surface reactions of the propionic acid conversion to ethane and ethylene over a $\mathrm{Ni}$ (111) catalyst surface model at two different temperature of $298 \mathrm{~K}$ and $473 \mathrm{~K}$ based on two different implicit solvation schemes: iSMS and VASPsol. $\Delta \Delta \mathrm{G}^{\text {act }}$ indicates the activation free energy differences between corresponding reaction in liquid water and in the gas phase.

\begin{tabular}{|c|c|c|c|c|c|}
\hline \multirow{3}{*}{ \# } & \multirow{3}{*}{ Reaction } & \multicolumn{4}{|c|}{$\Delta \Delta \mathbf{G}^{\text {act }}$} \\
\hline & & \multicolumn{2}{|c|}{$T=298 \mathrm{~K}$} & \multicolumn{2}{|c|}{$\mathrm{T}=473 \mathrm{~K}$} \\
\hline & & iSMS & VASPsol & iSMS & VASPsol \\
\hline 1 & $\mathrm{CH}_{3} \mathrm{CH}_{2} \mathrm{COOH}^{*}+3^{*} \rightarrow \mathrm{CH}_{3} \mathrm{CH}_{2} \mathrm{CO}^{\star * *}+\mathrm{OH}^{*}$ & 0.04 & 0.29 & 0.00 & 0.29 \\
\hline 2 & $\mathrm{CH}_{3} \mathrm{CH}_{2} \mathrm{COOH}^{\star}+2^{*} \rightarrow \mathrm{CH}_{3} \mathrm{CHCOOH}^{\star *}+\mathrm{H}^{*}$ & -0.03 & 0.05 & -0.03 & 0.03 \\
\hline 3 & $\mathrm{CH}_{3} \mathrm{CH}_{2} \mathrm{CO}^{\star \star \star}+2^{\star} \rightarrow \mathrm{CH}_{3} \mathrm{CH}_{2}{ }^{\star \star}+\mathrm{CO}^{\star \star \star}$ & -0.02 & 0.01 & -0.03 & 0.02 \\
\hline 4 & $\mathrm{CH}_{3} \mathrm{CH}_{2} \mathrm{CO}^{\star \star *} \rightarrow \mathrm{CH}_{3} \mathrm{CHCO}^{\star *}+\mathrm{H}^{*}$ & -0.03 & 0.01 & -0.03 & 0.05 \\
\hline 5 & $\mathrm{CH}_{3} \mathrm{CHCOOH}^{* *}+{ }^{*} \rightarrow \mathrm{CH}_{3} \mathrm{CHCO}^{* *}+\mathrm{OH}^{*}$ & -0.02 & 0.13 & -0.03 & 0.13 \\
\hline 6 & $\mathrm{CH}_{3} \mathrm{CHCOOH}^{\star *}+3^{*} \rightarrow \mathrm{CH}_{2} \mathrm{CHCOOH}^{\star * \star *}+\mathrm{H}^{*}$ & -0.01 & -0.06 & 0.00 & -0.04 \\
\hline 7 & $\mathrm{CH}_{3} \mathrm{CHCOOH}^{\star \star}+2^{\star} \rightarrow \mathrm{CH}_{3} \mathrm{CCOOH}^{\star \star \star}+\mathrm{H}^{*}$ & 0.00 & 0.00 & 0.00 & 0.02 \\
\hline 8 & $\mathrm{CH}_{3} \mathrm{CHCO}^{\star \star}+4^{*} \rightarrow \mathrm{CH}_{3} \mathrm{CH}^{\star \star \star}+\mathrm{CO}^{\star \star *}$ & -0.01 & 0.00 & -0.01 & 0.00 \\
\hline 9 & $\mathrm{CH}_{3} \mathrm{CHCO}^{\star \star}+3^{\star} \rightarrow \mathrm{CH}_{3} \mathrm{CCO}^{\star \star \star \star}+\mathrm{H}^{*}$ & -0.08 & 0.04 & -0.06 & 0.07 \\
\hline 10 & $\mathrm{CH}_{3} \mathrm{CHCO}^{\star \star}+3^{\star} \rightarrow \mathrm{CH}_{2} \mathrm{CHCO}^{\star \star \star \star}+\mathrm{H}^{\star}$ & 0.01 & -0.02 & 0.00 & 0.01 \\
\hline 11 & $\mathrm{CH}_{2} \mathrm{CHCOOH}^{\star \star * \star}+{ }^{*} \rightarrow \mathrm{CH}_{2} \mathrm{CHCO}^{\star \star * *}+\mathrm{OH}^{*}$ & 0.03 & 0.15 & 0.00 & 0.13 \\
\hline 12 & $\mathrm{CH}_{2} \mathrm{CHCOOH}^{\star \star \star \star}+{ }^{*} \rightarrow \mathrm{CHCHCOOH} H^{\star \star \star \star}+\mathrm{H}^{*}$ & 0.01 & 0.00 & 0.01 & -0.01 \\
\hline 13 & $\mathrm{CH}_{3} \mathrm{CCOOH}^{\star \star *}+2^{*} \rightarrow \mathrm{CH}_{3} \mathrm{CCO}^{\star \star * *}+\mathrm{OH}^{*}$ & -0.06 & 0.10 & -0.08 & 0.09 \\
\hline 14 & $\mathrm{CH}_{3} \mathrm{CCO}^{\star \star \star *}+2^{*} \rightarrow \mathrm{CH}_{3} \mathrm{C}^{\star \star *}+\mathrm{CO}^{\star \star \star}$ & -0.08 & 0.21 & -0.08 & 0.18 \\
\hline 15 & $\mathrm{CH}_{2} \mathrm{CHCO}^{\star \star * *}+2^{*} \rightarrow \mathrm{CH}_{2} \mathrm{CH}^{* \star *}+\mathrm{CO}^{\star \star *}$ & 0.00 & -0.04 & -0.01 & -0.05 \\
\hline 16 & $\mathrm{CH}_{2} \mathrm{CHCO}^{\star \star * *}+* \rightarrow \mathrm{CHCHCO}{ }^{\star * \star *}+\mathrm{H}^{*}$ & 0.00 & -0.04 & 0.00 & -0.05 \\
\hline 17 & $\mathrm{CHCHCOOH}^{\star * \star *}+* \rightarrow \mathrm{CHCHCO}{ }^{* \star * *}+\mathrm{OH}^{*}$ & -0.11 & 0.14 & -0.12 & 0.12 \\
\hline 18 & $\mathrm{CHCHCO}^{\star \star \star \star}+3^{\star} \rightarrow \mathrm{CHCH}^{\star \star \star \star}+\mathrm{CO}^{\star \star \star}$ & -0.02 & 0.00 & -0.03 & 0.00 \\
\hline 19 & $\mathrm{CH}_{2} \mathrm{CH}^{\star \star \star}+2^{\star} \rightarrow \mathrm{CHCH}^{\star \star \star \star}+\mathrm{H}^{*}$ & 0.02 & -0.01 & 0.02 & 0.00 \\
\hline 20 & $\mathrm{CH}_{2} \mathrm{CH}_{2}{ }^{\star \star}+2^{\star} \rightarrow \mathrm{CH}_{2} \mathrm{CH}^{\star \star \star}+\mathrm{H}^{\star}$ & 0.01 & -0.02 & 0.01 & -0.01 \\
\hline 21 & $\mathrm{CH}_{2} \mathrm{CH}^{\star \star \star}+* \rightarrow \mathrm{CH}_{2} \mathrm{C}^{\star \star \star}+\mathrm{H}^{\star}$ & -0.02 & 0.03 & -0.02 & 0.02 \\
\hline 22 & $\mathrm{CH}_{3} \mathrm{C}^{\star \star *}+{ }^{*} \rightarrow \mathrm{CH}_{2} \mathrm{C}^{\star \star \star}+\mathrm{H}^{\star}$ & -0.01 & -0.04 & -0.01 & -0.04 \\
\hline 23 & $\mathrm{CH}_{3} \mathrm{CH}^{\star \star \star}+{ }^{*} \rightarrow \mathrm{CH}_{2} \mathrm{CH}^{\star \star \star}+\mathrm{H}^{*}$ & -0.02 & -0.05 & -0.03 & -0.06 \\
\hline 24 & $\mathrm{CH}_{3} \mathrm{CH}^{\star \star \star}+* \rightarrow \mathrm{CH}_{3} \mathrm{C}^{\star \star \star}+\mathrm{H}^{\star}$ & 0.02 & 0.00 & 0.02 & -0.01 \\
\hline 25 & $\mathrm{CH}_{3} \mathrm{CH}_{2}{ }^{\star \star}+2^{\star} \rightarrow \mathrm{CH}_{3} \mathrm{CH}^{\star \star \star}+\mathrm{H}^{\star}$ & 0.02 & 0.02 & 0.01 & 0.03 \\
\hline 26 & $\mathrm{CH}_{3} \mathrm{CH}_{3}{ }^{*}+2^{*} \rightarrow \mathrm{CH}_{3} \mathrm{CH}_{2}{ }^{\star *}+\mathrm{H}^{*}$ & -0.01 & -0.04 & -0.02 & -0.01 \\
\hline 27 & $\mathrm{CH}_{3} \mathrm{CH}_{2}{ }^{\star \star}+{ }^{*} \rightarrow \mathrm{CH}_{2} \mathrm{CH}_{2}{ }^{\star *}+\mathrm{H}^{*}$ & 0.00 & -0.07 & -0.01 & -0.06 \\
\hline 28 & $\mathrm{CH}_{3} \mathrm{CH}_{2} \mathrm{COOH}^{*}+2^{*} \rightarrow \mathrm{CH}_{3} \mathrm{CH}_{2} \mathrm{COO}^{\star *}+\mathrm{H}^{*}$ & -0.04 & 0.29 & -0.07 & 0.24 \\
\hline 29 & $\mathrm{CH}_{3} \mathrm{CH}_{2} \mathrm{COO}^{\star \star}+{ }^{*} \rightarrow \mathrm{CH}_{3} \mathrm{CH}_{2}^{* \star}+\mathrm{CO}_{2}{ }^{*}$ & -0.01 & -0.01 & -0.04 & 0.01 \\
\hline 30 & $\mathrm{CH}_{3} \mathrm{CH}_{2} \mathrm{COO}^{\star \star}+2^{\star} \rightarrow \mathrm{CH}_{3} \mathrm{CHCOO}^{\star \star \star}+\mathrm{H}^{\star}$ & -0.19 & -0.01 & -0.17 & -0.01 \\
\hline 31 & $\mathrm{CH}_{3} \mathrm{CHCOOH}^{\star \star}+2^{*} \rightarrow \mathrm{CH}_{3} \mathrm{CHCOO}^{\star \star \star}+\mathrm{H}^{*}$ & -0.14 & 0.21 & -0.14 & 0.19 \\
\hline 32 & $\mathrm{CH}_{3} \mathrm{CHCOOH}^{\star \star}+3^{\star} \rightarrow \mathrm{CH}_{3} \mathrm{CH}^{\star \star \star}+\mathrm{COOH}^{\star \star}$ & -0.02 & 0.01 & -0.02 & 0.05 \\
\hline 33 & $\mathrm{CH}_{3} \mathrm{CHCOO}^{\star \star \star}+{ }^{*} \rightarrow \mathrm{CH}_{3} \mathrm{CH}^{\star \star \star}+\mathrm{CO}_{2}{ }^{*}$ & 0.16 & 0.01 & 0.12 & 0.00 \\
\hline 34 & $\mathrm{CH}_{3} \mathrm{CHCOO}^{* \star *}+{ }^{*} \rightarrow \mathrm{CH}_{3} \mathrm{CCOO}^{\star * *}+\mathrm{H}^{*}$ & 0.14 & 0.03 & 0.12 & 0.03 \\
\hline 35 & $\mathrm{CH}_{3} \mathrm{CCOOH}^{* * *}+* \rightarrow \mathrm{CH}_{3} \mathrm{CCOO}^{* * *}+\mathrm{H}^{*}$ & -0.20 & 0.12 & -0.18 & 0.11 \\
\hline 36 & $\mathrm{CH}_{3} \mathrm{CCOOH}^{\star \star \star}+2^{\star} \rightarrow \mathrm{CH}_{3} \mathrm{C}^{\star \star \star}+\mathrm{COOH}^{\star \star}$ & -0.04 & -0.01 & -0.05 & 0.01 \\
\hline 37 & $\mathrm{CH}_{2} \mathrm{CHCOOH}^{\star \star \star \star}+* \rightarrow \mathrm{CH}_{2} \mathrm{CH}^{\star \star \star}+\mathrm{COOH}^{\star \star}$ & 0.00 & 0.02 & -0.01 & 0.03 \\
\hline 38 & $\mathrm{CH}_{3} \mathrm{CCOO}^{\star \star *}+{ }^{*} \rightarrow \mathrm{CH}_{3} \mathrm{C}^{\star * *}+\mathrm{CO}_{2}{ }^{*}$ & 0.28 & 0.13 & 0.21 & 0.11 \\
\hline 39 & $\mathrm{COOH}^{\star \star} \rightarrow \mathrm{CO}_{2}^{*}+\mathrm{H}^{\star}$ & -0.15 & 0.08 & -0.18 & 0.06 \\
\hline 40 & $\mathrm{COOH}^{\star *}+2^{*} \rightarrow \mathrm{CO}^{\star \star *}+\mathrm{OH}^{*}$ & 0.06 & -0.01 & 0.04 & -0.02 \\
\hline 41 & $\mathrm{H}_{2} \mathrm{O}^{*}+{ }^{*} \rightarrow \mathrm{OH}^{*}+\mathrm{H}^{*}$ & 0.00 & -0.22 & -0.01 & -0.33 \\
\hline
\end{tabular}


1 Table S5. Overall turnover frequency of hydrodeoxygenation of propionic acid over a Ni (111) catalyst surface model

2 at a temperature of $473 \mathrm{~K}$ in vapor phase, and at different partial pressures of hydrogen and carbon monoxide.

\begin{tabular}{cccccc}
\hline $\mathbf{P}_{\mathrm{co}} \backslash \mathbf{P}_{\mathrm{H} 2}$ (bar) & $\mathbf{0 . 0 1}$ & $\mathbf{0 . 1}$ & $\mathbf{1}$ & $\mathbf{1 0}$ & $\mathbf{3 0}$ \\
\hline $\mathbf{0 . 0 0 0 1}$ & $3.99 \times 10^{-6}$ & $1.98 \times 10^{-8}$ & $8.72 \times 10^{-11}$ & $7.23 \times 10^{-13}$ & $7.72 \times 10^{-14}$ \\
$\mathbf{0 . 0 0 1}$ & $2.66 \times 10^{-6}$ & $3.46 \times 10^{-8}$ & $1.30 \times 10^{-10}$ & $7.41 \times 10^{-13}$ & $7.76 \times 10^{-14}$ \\
$\mathbf{0 . 0 1}$ & $6.92 \times 10^{-7}$ & $1.63 \times 10^{-8}$ & $1.86 \times 10^{-10}$ & $8.76 \times 10^{-13}$ & $8.10 \times 10^{-14}$ \\
$\mathbf{0 . 1}$ & $8.98 \times 10^{-8}$ & $5.09 \times 10^{-9}$ & $9.26 \times 10^{-11}$ & $1.20 \times 10^{-12}$ & $1.02 \times 10^{-13}$ \\
$\mathbf{1}$ & $5.72 \times 10^{-9}$ & $9.47 \times 10^{-10}$ & $3.30 \times 10^{-11}$ & $8.55 \times 10^{-13}$ & $1.24 \times 10^{-13}$ \\
\hline
\end{tabular}

3

Table S6. Overall turnover frequency of hydrodeoxygenation of propionic acid over a $\mathrm{Ni}$ (111) catalyst surface model at a temperature of $473 \mathrm{~K}$ in vapor phase, and at different partial pressures of hydrogen and carbon monoxide based on considering only 1 site for adsorbed CO and analogues change in counting sites for other adsorbates attached to the surface through their carbon atom. The number of sites assigned to each adsorbate in this calculation is included in Table S7.

\begin{tabular}{cccccc}
\hline $\mathbf{P}_{\mathrm{CO}} \backslash \mathbf{P}_{\mathrm{H} 2}$ (bar) & $\mathbf{0 . 0 1}$ & $\mathbf{0 . 1}$ & $\mathbf{1}$ & $\mathbf{1 0}$ & $\mathbf{3 0}$ \\
\hline $\mathbf{0 . 0 0 0 1}$ & $1.14 \times 10^{-8}$ & $1.02 \times 10^{-8}$ & $6.12 \times 10^{-9}$ & $1.77 \times 10^{-9}$ & $6.63 \times 10^{-10}$ \\
$\mathbf{0 . 0 0 1}$ & $9.91 \times 10^{-12}$ & $9.61 \times 10^{-12}$ & $8.87 \times 10^{-12}$ & $6.99 \times 10^{-12}$ & $5.54 \times 10^{-12}$ \\
$\mathbf{0 . 0 1}$ & $8.50 \times 10^{-15}$ & $7.46 \times 10^{-15}$ & $7.07 \times 10^{-15}$ & $6.74 \times 10^{-15}$ & $6.50 \times 10^{-15}$ \\
$\mathbf{0 . 1}$ & $2.99 \times 10^{-17}$ & $1.27 \times 10^{-17}$ & $7.33 \times 10^{-18}$ & $5.59 \times 10^{-18}$ & $5.24 \times 10^{-18}$ \\
$\mathbf{1}$ & $4.20 \times 10^{-19}$ & $1.35 \times 10^{-19}$ & $4.49 \times 10^{-20}$ & $1.64 \times 10^{-20}$ & $1.09 \times 10^{-20}$ \\
\hline
\end{tabular}

9 
1 Table S7. Number of occupied sites used for microkinetic modeling result displayed in Table S6.

\begin{tabular}{|c|c|}
\hline Adsorbed Species & \# of occupied site \\
\hline $\mathrm{CH}_{2} \mathrm{C}$ & 2 \\
\hline $\mathrm{CH}_{2} \mathrm{CH}$ & 3 \\
\hline $\mathrm{CH}_{2} \mathrm{CH}$ & 2 \\
\hline $\mathrm{CH}_{2} \mathrm{CHCO}$ & 3 \\
\hline $\mathrm{CH}_{2} \mathrm{CHCOOH}$ & 3 \\
\hline $\mathrm{CH}_{3} \mathrm{C}$ & 1 \\
\hline $\mathrm{CH}_{3} \mathrm{CCO}$ & 3 \\
\hline $\mathrm{CH}_{3} \mathrm{CCOO}$ & 3 \\
\hline $\mathrm{CH}_{3} \mathrm{CCOOH}$ & 3 \\
\hline $\mathrm{CH}_{3} \mathrm{CH}$ & 2 \\
\hline $\mathrm{CH}_{3} \mathrm{CH}_{2}$ & 1 \\
\hline $\mathrm{CH}_{3} \mathrm{CH}_{2} \mathrm{CO}$ & 3 \\
\hline $\mathrm{CH}_{3} \mathrm{CH}_{2} \mathrm{COO}$ & 2 \\
\hline $\mathrm{CH}_{3} \mathrm{CH}_{2} \mathrm{COOH}$ & 1 \\
\hline $\mathrm{CH}_{3} \mathrm{CH}_{3}$ & 1 \\
\hline $\mathrm{CH}_{3} \mathrm{CHCO}$ & 2 \\
\hline $\mathrm{CH}_{3} \mathrm{CHCOO}$ & 3 \\
\hline $\mathrm{CH}_{3} \mathrm{CHCOOH}$ & 2 \\
\hline $\mathrm{CHCH}$ & 3 \\
\hline $\mathrm{CHCHCO}$ & 4 \\
\hline $\mathrm{CHCHCOOH}$ & 3 \\
\hline $\mathrm{CO}$ & 1 \\
\hline $\mathrm{CO}_{2}$ & 1 \\
\hline $\mathrm{COOH}$ & 2 \\
\hline $\mathrm{H}$ & 1 \\
\hline $\mathrm{H}_{2} \mathrm{O}$ & 1 \\
\hline $\mathrm{OH}$ & 1 \\
\hline
\end{tabular}

2 
1

Table S8. Solvent effect on the stability of various adsorbed species in the propionic acid conversion to ethane and ethylene over a $\mathrm{Ni}(111)$ catalyst surface model at $473 \mathrm{~K}$ in the presence of two different solvents, water and 1,4dioxane, using iSMS methodology. $\Delta \Delta \mathrm{G}_{\mathrm{rxn}}$ indicates the difference in the adsorption free energy of the corresponding intermediates in the presence and absence of solvent. Asterisk (*) represents a surface adsorption site and multiple asterisks are indicative of the number of occupied active sites. Note that calculations for solvents were performed with the help of the COSMO-RS package with three different Ni cavity radii: with default value, with a $10 \%$ increased value and a $10 \%$ decreased value relative to the default.

\begin{tabular}{|c|c|c|c|c|c|c|}
\hline \multirow{3}{*}{ Adsorbed species } & \multicolumn{6}{|c|}{$\Delta \Delta G_{r \times n}, e V @ 473 K$} \\
\hline & \multicolumn{3}{|c|}{ Water } & \multicolumn{3}{|c|}{ 1,4-dioxane } \\
\hline & Default & $+10 \%$ & $-10 \%$ & Default & $+10 \%$ & $-10 \%$ \\
\hline $\mathrm{CH}_{2} \mathrm{C}^{\star * *}$ & -0.08 & -0.09 & -0.06 & -0.05 & -0.04 & -0.03 \\
\hline $\mathrm{CH}_{2} \mathrm{CH}^{* \star *}$ & -0.01 & -0.03 & 0.05 & 0.00 & 0.00 & 0.05 \\
\hline $\mathrm{CH}_{2} \mathrm{CH}_{2}{ }^{\star \star}$ & 0.10 & 0.02 & 0.20 & 0.12 & 0.06 & 0.18 \\
\hline $\mathrm{CH}_{2} \mathrm{CHCO}^{\star \star \star \star}$ & -0.09 & -0.13 & -0.04 & -0.03 & -0.04 & 0.01 \\
\hline $\mathrm{CH}_{2} \mathrm{CHCOOH}^{\star \star * *}$ & -0.11 & -0.16 & -0.03 & -0.04 & -0.07 & 0.01 \\
\hline $\mathrm{CH}_{3} \mathrm{C}^{\star \star *}$ & -0.01 & -0.03 & 0.03 & -0.01 & 0.00 & 0.02 \\
\hline $\mathrm{CH}_{3} \mathrm{CCO}^{\star \star \star \star *}$ & -0.12 & -0.15 & -0.09 & -0.06 & -0.06 & -0.04 \\
\hline $\mathrm{CH}_{3} \mathrm{CCOO}^{\star * *}$ & -0.55 & -0.51 & -0.58 & -0.33 & -0.27 & -0.36 \\
\hline $\mathrm{CH}_{3} \mathrm{CCOOH}^{\star \star *}$ & -0.12 & -0.17 & -0.08 & -0.07 & -0.09 & -0.04 \\
\hline $\mathrm{CH}_{3} \mathrm{CH}^{\star \star *}$ & 0.00 & -0.02 & 0.07 & -0.01 & 0.01 & 0.05 \\
\hline $\mathrm{CH}_{3} \mathrm{CH}_{2}{ }^{\star \star}$ & 0.01 & -0.02 & 0.07 & -0.01 & 0.00 & 0.03 \\
\hline $\mathrm{CH}_{3} \mathrm{CH}_{2} \mathrm{CO}^{\star \star *}$ & -0.05 & -0.08 & 0.01 & -0.04 & -0.04 & 0.00 \\
\hline $\mathrm{CH}_{3} \mathrm{CH}_{2} \mathrm{COO}^{* *}$ & -0.06 & -0.09 & -0.03 & -0.04 & -0.04 & -0.04 \\
\hline $\mathrm{CH}_{3} \mathrm{CH}_{2} \mathrm{COOH}^{*}$ & -0.03 & -0.11 & 0.06 & 0.02 & -0.04 & 0.08 \\
\hline $\mathrm{CH}_{3} \mathrm{CH}_{3}{ }^{*}$ & 0.02 & -0.02 & 0.07 & 0.02 & -0.01 & 0.04 \\
\hline $\mathrm{CH}_{3} \mathrm{CHCO}^{* *}$ & -0.09 & -0.13 & -0.05 & -0.05 & -0.06 & -0.02 \\
\hline $\mathrm{CH}_{3} \mathrm{CHCOO}^{\star \star *}$ & -0.31 & -0.30 & -0.33 & -0.17 & -0.13 & -0.18 \\
\hline $\mathrm{CH}_{3} \mathrm{CHCOOH}^{\star *}$ & -0.10 & -0.15 & -0.04 & -0.06 & -0.07 & -0.03 \\
\hline $\mathrm{CHCH}^{\star \star \star *}$ & 0.05 & -0.01 & 0.13 & 0.08 & 0.05 & 0.13 \\
\hline $\mathrm{CHCHCO} * * * *$ & -0.15 & -0.19 & -0.14 & -0.05 & -0.08 & -0.05 \\
\hline $\mathrm{CHCHCOOH}^{* \star * *}$ & -0.13 & -0.19 & -0.09 & -0.06 & -0.09 & -0.03 \\
\hline $\mathrm{CO}^{* * *}$ & -0.25 & -0.25 & -0.25 & -0.18 & -0.16 & -0.20 \\
\hline $\mathrm{CO}_{2}{ }^{*}$ & -0.04 & -0.08 & 0.04 & 0.01 & -0.02 & 0.04 \\
\hline $\mathrm{COOH}^{* *}$ & -0.16 & -0.17 & -0.13 & -0.08 & -0.07 & -0.05 \\
\hline $\mathrm{H}^{*}$ & -0.01 & 0.02 & 0.03 & 0.00 & 0.03 & 0.03 \\
\hline $\mathrm{H}_{2} \mathrm{O}$ * & 0.02 & -0.03 & 0.09 & 0.07 & 0.03 & 0.13 \\
\hline $\mathrm{OH}^{*}$ & 0.00 & -0.03 & 0.06 & 0.02 & 0.01 & 0.08 \\
\hline
\end{tabular}


1 Table S9. Solvent effect on the reaction free energies of all elementary surface reactions of the propionic acid 2 conversion to ethane and ethylene over a $\mathrm{Ni}$ (111) catalyst surface model at $473 \mathrm{~K}$ based on iSMS methodology in the 3 presence of water and 1,4-dioxane. $\Delta \Delta \mathrm{G}_{\text {rxn }}$ indicates the reaction free energy difference between corresponding 4 reaction in solvent and in a gas phase. Note that calculations for solvents were performed with the help of the COSMO5 RS package with three different Ni cavity radii: with default value, with a $10 \%$ increased value and a $10 \%$ decreased 6 value relative to the default.

\begin{tabular}{|c|c|c|c|c|c|c|c|}
\hline \multirow{3}{*}{ \# } & \multirow{3}{*}{ Reaction } & \multicolumn{6}{|c|}{$\Delta \Delta G_{r x n}, e V @ 473 K$} \\
\hline & & \multicolumn{3}{|c|}{ Water } & \multicolumn{3}{|c|}{ 1,4-dioxane } \\
\hline & & Default & $+10 \%$ & $-10 \%$ & Default & $+10 \%$ & $-10 \%$ \\
\hline 1 & $\mathrm{CH}_{3} \mathrm{CH}_{2} \mathrm{COOH}^{\star}+3^{*} \rightarrow \mathrm{CH}_{3} \mathrm{CH}_{2} \mathrm{CO}^{\star \star *}+\mathrm{OH}^{*}$ & 0.00 & 0.01 & 0.05 & 0.03 & 0.04 & 0.08 \\
\hline 2 & $\mathrm{CH}_{3} \mathrm{CH}_{2} \mathrm{COOH}^{*}+2^{*} \rightarrow \mathrm{CH}_{3} \mathrm{CHCOOH}^{* *}+\mathrm{H}^{*}$ & -0.07 & -0.04 & -0.07 & -0.03 & 0.00 & -0.03 \\
\hline 3 & $\mathrm{CH}_{3} \mathrm{CH}_{2} \mathrm{CO}^{* * *}+2^{*} \rightarrow \mathrm{CH}_{3} \mathrm{CH}_{2}{ }^{* *}+\mathrm{CO}^{* * *}$ & -0.14 & -0.12 & -0.18 & -0.10 & -0.07 & -0.15 \\
\hline 4 & $\mathrm{CH}_{3} \mathrm{CH}_{2} \mathrm{CO}^{\star * *} \rightarrow \mathrm{CH}_{3} \mathrm{CHCO}^{* *}+\mathrm{H}^{*}$ & -0.06 & -0.07 & -0.07 & -0.02 & -0.02 & -0.03 \\
\hline 5 & $\mathrm{CH}_{3} \mathrm{CHCOOH}^{\star *}+* \rightarrow \mathrm{CH}_{3} \mathrm{CHCO}^{\star \star}+\mathrm{OH}^{*}$ & 0.01 & -0.02 & 0.05 & 0.04 & 0.02 & 0.09 \\
\hline 6 & $\mathrm{CH}_{3} \mathrm{CHCOOH}^{* *}+3^{\star} \rightarrow \mathrm{CH}_{2} \mathrm{CHCOOH}^{\star * * *}+\mathrm{H}^{*}$ & -0.02 & -0.02 & 0.00 & 0.01 & 0.01 & 0.02 \\
\hline 7 & $\mathrm{CH}_{3} \mathrm{CHCOOH}^{\star \star}+2^{\star} \rightarrow \mathrm{CH}_{3} \mathrm{CCOOH}^{\star * *}+\mathrm{H}^{*}$ & -0.03 & -0.03 & -0.06 & -0.01 & -0.02 & -0.03 \\
\hline 8 & $\mathrm{CH}_{3} \mathrm{CHCO}^{\star \star}+4^{\star} \rightarrow \mathrm{CH}_{3} \mathrm{CH}^{\star \star \star}+\mathrm{CO}^{\star \star *}$ & -0.10 & -0.07 & -0.14 & -0.09 & -0.04 & -0.12 \\
\hline 9 & $\mathrm{CH}_{3} \mathrm{CHCO}^{* *}+3^{*} \rightarrow \mathrm{CH}_{3} \mathrm{CCO}^{* * *}+\mathrm{H}^{*}$ & -0.05 & -0.03 & -0.06 & -0.02 & 0.00 & -0.03 \\
\hline 10 & $\mathrm{CH}_{3} \mathrm{CHCO}^{\star *}+3^{*} \rightarrow \mathrm{CH}_{2} \mathrm{CHCO}^{\star * * *}+\mathrm{H}^{*}$ & -0.01 & 0.00 & -0.01 & 0.01 & 0.02 & 0.01 \\
\hline 11 & $\mathrm{CH}_{2} \mathrm{CHCOOH}^{* \star * *}+{ }^{*} \rightarrow \mathrm{CH}_{2} \mathrm{CHCO}^{\star * \star *}+\mathrm{OH}^{*}$ & 0.02 & 0.00 & 0.04 & 0.04 & 0.04 & 0.08 \\
\hline 12 & $\mathrm{CH}_{2} \mathrm{CHCOOH}^{\star \star \star \star}+{ }^{*} \rightarrow \mathrm{CHCHCOOH} H^{\star \star \star \star}+\mathrm{H}^{\star}$ & -0.05 & -0.04 & -0.07 & -0.03 & -0.03 & -0.04 \\
\hline 13 & $\mathrm{CH}_{3} \mathrm{CCOOH}^{* \star *}+2^{*} \rightarrow \mathrm{CH}_{3} \mathrm{CCO}^{\star * \star *}+\mathrm{OH}^{*}$ & -0.01 & -0.02 & 0.04 & 0.03 & 0.04 & 0.09 \\
\hline 14 & $\mathrm{CH}_{3} \mathrm{CCO}^{* \star *}+2^{*} \rightarrow \mathrm{CH}_{3} \mathrm{C}^{\star * *}+\mathrm{CO}^{* * *}$ & -0.08 & -0.06 & -0.13 & -0.08 & -0.05 & -0.12 \\
\hline 15 & $\mathrm{CH}_{2} \mathrm{CHCO}^{\star \star \star *}+2^{\star} \rightarrow \mathrm{CH}_{2} \mathrm{CH}^{\star \star \star}+\mathrm{CO}^{\star \star *}$ & -0.11 & -0.09 & -0.15 & -0.10 & -0.07 & -0.14 \\
\hline 16 & $\mathrm{CH}_{2} \mathrm{CHCO}^{\star \star \star \star *}+{ }^{*} \rightarrow \mathrm{CHCHCO}{ }^{\star \star \star *}+\mathrm{H}^{\star}$ & -0.08 & -0.08 & -0.11 & -0.03 & -0.04 & -0.07 \\
\hline 17 & $\mathrm{CHCHCOOH}^{\star \star * *}+* \rightarrow \mathrm{CHCHCO}{ }^{* \star * \star}+\mathrm{OH}^{*}$ & -0.01 & -0.04 & 0.00 & 0.03 & 0.02 & 0.06 \\
\hline 18 & $\mathrm{CHCHCO}^{\star \star * *}+3^{*} \rightarrow \mathrm{CHCH}^{* \star \star \star}+\mathrm{CO}^{\star \star *}$ & -0.06 & -0.04 & -0.08 & -0.08 & -0.03 & -0.09 \\
\hline 19 & $\mathrm{CH}_{2} \mathrm{CH}^{\star \star \star}+2^{*} \rightarrow \mathrm{CHCH}^{\star \star \star \star}+\mathrm{H}^{\star}$ & -0.03 & -0.03 & -0.03 & -0.01 & 0.00 & -0.02 \\
\hline 20 & $\mathrm{CH}_{2} \mathrm{CH}_{2}{ }^{\star \star}+2^{\star} \rightarrow \mathrm{CH}_{2} \mathrm{CH}^{\star \star \star}+\mathrm{H}^{\star}$ & -0.06 & -0.05 & -0.09 & -0.03 & -0.02 & -0.06 \\
\hline 21 & $\mathrm{CH}_{2} \mathrm{CH}^{\star \star *}+* \rightarrow \mathrm{CH}_{2} \mathrm{C}^{\star * *}+\mathrm{H}^{*}$ & -0.09 & -0.08 & -0.13 & -0.06 & -0.05 & -0.09 \\
\hline 22 & $\mathrm{CH}_{3} \mathrm{C}^{\star \star \star}+{ }^{*} \rightarrow \mathrm{CH}_{2} \mathrm{C}^{\star \star *}+\mathrm{H}^{*}$ & -0.08 & -0.07 & -0.11 & -0.05 & -0.04 & -0.06 \\
\hline 23 & $\mathrm{CH}_{3} \mathrm{CH}^{\star \star \star}+* \rightarrow \mathrm{CH}_{2} \mathrm{CH}^{\star \star \star}+\mathrm{H}^{*}$ & -0.03 & -0.02 & -0.03 & -0.01 & 0.00 & -0.01 \\
\hline 24 & $\mathrm{CH}_{3} \mathrm{CH}^{\star \star \star}+{ }^{*} \rightarrow \mathrm{CH}_{3} \mathrm{C}^{\star \star *}+\mathrm{H}^{*}$ & -0.03 & -0.02 & -0.05 & -0.01 & -0.01 & -0.04 \\
\hline 25 & $\mathrm{CH}_{3} \mathrm{CH}_{2}{ }^{* *}+2^{*} \rightarrow \mathrm{CH}_{3} \mathrm{CH}^{\star \star *}+\mathrm{H}^{*}$ & -0.03 & -0.02 & -0.02 & -0.01 & 0.01 & 0.01 \\
\hline 26 & $\mathrm{CH}_{3} \mathrm{CH}_{3}{ }^{*}+2^{\star} \rightarrow \mathrm{CH}_{3} \mathrm{CH}_{2}{ }^{* *}+\mathrm{H}^{*}$ & -0.05 & -0.03 & -0.05 & -0.03 & 0.00 & -0.02 \\
\hline 27 & $\mathrm{CH}_{3} \mathrm{CH}_{2}{ }^{\star *}+* \rightarrow \mathrm{CH}_{2} \mathrm{CH}_{2}{ }^{\star *}+\mathrm{H}^{\star}$ & 0.01 & 0.01 & 0.04 & 0.02 & 0.02 & 0.05 \\
\hline 28 & $\mathrm{CH}_{3} \mathrm{CH}_{2} \mathrm{COOH}^{*}+2^{*} \rightarrow \mathrm{CH}_{3} \mathrm{CH}_{2} \mathrm{COO}^{\star *}+\mathrm{H}^{*}$ & -0.03 & 0.02 & -0.06 & 0.00 & 0.04 & -0.05 \\
\hline 29 & $\mathrm{CH}_{3} \mathrm{CH}_{2} \mathrm{COO}^{* *}+{ }^{*} \rightarrow \mathrm{CH}_{3} \mathrm{CH}_{2}^{* *}+\mathrm{CO}_{2}^{*}$ & 0.06 & 0.03 & 0.17 & 0.04 & 0.03 & 0.13 \\
\hline 30 & $\mathrm{CH}_{3} \mathrm{CH} 2 \mathrm{COO}^{\star \star}+2^{\star} \rightarrow \mathrm{CH}_{3} \mathrm{CHCOO}^{\star \star *}+\mathrm{H}^{*}$ & -0.27 & -0.22 & -0.32 & -0.14 & -0.10 & -0.15 \\
\hline 31 & $\mathrm{CH}_{3} \mathrm{CHCOOH}^{\star *}+2^{*} \rightarrow \mathrm{CH}_{3} \mathrm{CHCOO}^{\star * *}+\mathrm{H}^{*}$ & -0.23 & -0.17 & -0.30 & -0.11 & -0.06 & -0.17 \\
\hline 32 & $\mathrm{CH}_{3} \mathrm{CHCOOH}^{\star *}+3^{*} \rightarrow \mathrm{CH}_{3} \mathrm{CH}^{* * *}+\mathrm{COOH}^{\star *}$ & -0.06 & -0.05 & -0.02 & -0.02 & 0.00 & 0.03 \\
\hline 33 & $\mathrm{CH}_{3} \mathrm{CHCOO}^{* * *}+{ }^{*} \rightarrow \mathrm{CH}_{3} \mathrm{CH}^{\star * *}+\mathrm{CO}_{2}{ }^{*}$ & 0.31 & 0.24 & 0.47 & 0.17 & 0.13 & 0.29 \\
\hline 34 & $\mathrm{CH}_{3} \mathrm{CHCOO}^{* \star *}+* \rightarrow \mathrm{CH}_{3} \mathrm{CCOO}^{* * *}+\mathrm{H}^{*}$ & -0.26 & -0.23 & -0.27 & -0.17 & -0.14 & -0.19 \\
\hline 35 & $\mathrm{CH}_{3} \mathrm{CCOOH}^{\star \star \star}+* \rightarrow \mathrm{CH}_{3} \mathrm{CCOO}^{\star \star \star}+\mathrm{H}^{\star}$ & -0.45 & -0.36 & -0.51 & -0.27 & -0.19 & -0.32 \\
\hline 36 & $\mathrm{CH}_{3} \mathrm{CCOOH}^{\star \star \star}+2^{\star} \rightarrow \mathrm{CH}_{3} \mathrm{C}^{* \star \star}+\mathrm{COOH}^{\star \star}$ & -0.06 & -0.04 & -0.02 & -0.02 & 0.01 & 0.02 \\
\hline 37 & $\mathrm{CH}_{2} \mathrm{CHCOOH}^{\star \star \star \star}+* \rightarrow \mathrm{CH}_{2} \mathrm{CH}^{\star \star *}+\mathrm{COOH}^{\star \star}$ & -0.07 & -0.05 & -0.05 & -0.04 & -0.01 & -0.01 \\
\hline 38 & $\mathrm{CH}_{3} \mathrm{CCOO}^{\star * *}+* \rightarrow \mathrm{CH}_{3} \mathrm{C}^{\star * *}+\mathrm{CO}_{2}{ }^{*}$ & 0.54 & 0.44 & 0.68 & 0.33 & 0.26 & 0.44 \\
\hline 39 & $\mathrm{COOH}^{\star *} \rightarrow \mathrm{CO}_{2}^{*}+\mathrm{H}^{*}$ & 0.15 & 0.12 & 0.19 & 0.08 & 0.07 & 0.09 \\
\hline 40 & $\mathrm{COOH}^{\star *}+2^{*} \rightarrow \mathrm{CO}^{\star \star \star}+\mathrm{OH}^{*}$ & -0.03 & -0.04 & -0.06 & -0.03 & -0.03 & -0.05 \\
\hline 41 & $\mathrm{H}_{2} \mathrm{O}^{*}+* \rightarrow \mathrm{OH}^{*}+\mathrm{H}^{*}$ & 0.00 & 0.01 & 0.00 & 0.00 & 0.02 & 0.01 \\
\hline
\end{tabular}


Table S10. Solvent effect on the activation free energies of all elementary surface reactions of the propionic acid conversion to ethane and ethylene over a $\mathrm{Ni}$ (111) catalyst surface model at $473 \mathrm{~K}$ based on iSMS methodology in the presence of water and 1,4-dioxane. $\Delta \Delta \mathrm{G}^{\text {act }}$ indicates the activation free energy difference between corresponding reaction in solvent and in a gas phase. Note that calculations for solvents were performed with the help of the COSMORS package with three different Ni cavity radii: with default value, with a 10\% increased value and a 10\% decreased 6 value relative to the default.

\begin{tabular}{|c|c|c|c|c|c|c|c|}
\hline \multirow{3}{*}{$\#$} & \multirow{3}{*}{ Reaction } & \multicolumn{6}{|c|}{$\Delta \Delta G^{\text {act }}, \mathrm{eV} @ 473 \mathrm{~K}$} \\
\hline & & \multicolumn{3}{|c|}{ Water } & \multicolumn{3}{|c|}{ 1,4-dioxane } \\
\hline & & Default & $+10 \%$ & $-10 \%$ & Default & $+10 \%$ & $-10 \%$ \\
\hline 1 & $\mathrm{CH}_{3} \mathrm{CH}_{2} \mathrm{COOH}^{\star}+3^{*} \rightarrow \mathrm{CH}_{3} \mathrm{CH}_{2} \mathrm{CO}^{\star \star *}+\mathrm{OH}^{\star}$ & 0.00 & 0.03 & 0.02 & 0.00 & 0.01 & 0.02 \\
\hline 2 & $\mathrm{CH}_{3} \mathrm{CH}_{2} \mathrm{COOH}^{*}+2^{*} \rightarrow \mathrm{CH}_{3} \mathrm{CHCOOH}^{\star *}+\mathrm{H}^{*}$ & -0.03 & -0.01 & -0.02 & -0.01 & 0.01 & 0.00 \\
\hline 3 & $\mathrm{CH}_{3} \mathrm{CH}_{2} \mathrm{CO}^{\star * *}+2^{*} \rightarrow \mathrm{CH}_{3} \mathrm{CH}_{2}{ }^{* *}+\mathrm{CO}^{* * *}$ & -0.03 & -0.02 & -0.01 & 0.01 & 0.00 & 0.01 \\
\hline 4 & $\mathrm{CH}_{3} \mathrm{CH}_{2} \mathrm{CO}^{\star \star *} \rightarrow \mathrm{CH}_{3} \mathrm{CHCO}^{\star *}+\mathrm{H}^{\star}$ & -0.03 & -0.03 & -0.02 & 0.00 & 0.00 & 0.01 \\
\hline 5 & $\mathrm{CH}_{3} \mathrm{CHCOOH}^{* *}+* \rightarrow \mathrm{CH}_{3} \mathrm{CHCO}^{* *}+\mathrm{OH}^{*}$ & -0.03 & -0.03 & -0.05 & 0.00 & -0.01 & -0.01 \\
\hline 6 & $\mathrm{CH}_{3} \mathrm{CHCOOH}^{\star \star}+3^{\star} \rightarrow \mathrm{CH}_{2} \mathrm{CHCOOH}^{\star \star \star \star}+\mathrm{H}^{\star}$ & 0.00 & -0.01 & 0.02 & 0.04 & 0.02 & 0.05 \\
\hline 7 & $\mathrm{CH}_{3} \mathrm{CHCOOH}^{\star \star}+2^{\star} \rightarrow \mathrm{CH}_{3} \mathrm{CCOOH}^{\star \star \star}+\mathrm{H}^{\star}$ & 0.00 & 0.00 & 0.01 & 0.01 & 0.00 & 0.02 \\
\hline 8 & $\mathrm{CH}_{3} \mathrm{CHCO}^{\star *}+4^{\star} \rightarrow \mathrm{CH}_{3} \mathrm{CH}^{\star \star *}+\mathrm{CO}^{\star \star *}$ & -0.01 & 0.00 & -0.04 & 0.01 & 0.01 & -0.02 \\
\hline 9 & $\mathrm{CH}_{3} \mathrm{CHCO}^{\star *}+3^{\star} \rightarrow \mathrm{CH}_{3} \mathrm{CCO}^{\star \star \star *}+\mathrm{H}^{*}$ & -0.06 & -0.05 & -0.08 & -0.03 & -0.02 & -0.05 \\
\hline 10 & $\mathrm{CH}_{3} \mathrm{CHCO}^{\star \star}+3^{*} \rightarrow \mathrm{CH}_{2} \mathrm{CHCO}^{\star * \star *}+\mathrm{H}^{*}$ & 0.00 & 0.00 & -0.02 & 0.01 & 0.01 & 0.00 \\
\hline 11 & $\mathrm{CH}_{2} \mathrm{CHCOOH}^{\star \star \star *}+{ }^{*} \rightarrow \mathrm{CH}_{2} \mathrm{CHCO}^{\star \star \star *}+\mathrm{OH}^{\star}$ & 0.00 & 0.01 & -0.03 & 0.01 & 0.02 & -0.01 \\
\hline 12 & $\mathrm{CH}_{2} \mathrm{CHCOOH}^{\star \star \star \star}+{ }^{*} \rightarrow \mathrm{CHCHCOOH}^{\star \star \star \star}+\mathrm{H}^{\star}$ & 0.01 & 0.00 & 0.01 & 0.00 & -0.01 & 0.01 \\
\hline 13 & $\mathrm{CH}_{3} \mathrm{CCOOH}^{\star \star *}+2^{\star} \rightarrow \mathrm{CH}_{3} \mathrm{CCO}^{\star \star \star \star}+\mathrm{OH}^{*}$ & -0.08 & -0.05 & -0.08 & -0.03 & -0.01 & -0.02 \\
\hline 14 & $\mathrm{CH}_{3} \mathrm{CCO}^{\star \star \star \star}+2^{\star} \rightarrow \mathrm{CH}_{3} \mathrm{C}^{\star \star *}+\mathrm{CO}^{\star \star \star}$ & -0.08 & -0.06 & -0.11 & -0.07 & -0.05 & -0.09 \\
\hline 15 & $\mathrm{CH}_{2} \mathrm{CHCO}^{\star \star \star *}+2^{\star} \rightarrow \mathrm{CH}_{2} \mathrm{CH}^{\star * *}+\mathrm{CO}^{\star * *}$ & -0.01 & -0.01 & -0.01 & 0.00 & 0.00 & -0.01 \\
\hline 16 & $\mathrm{CH}_{2} \mathrm{CHCO}^{\star \star \star *}+{ }^{*} \rightarrow \mathrm{CHCHCO}{ }^{* \star \star *}+\mathrm{H}^{*}$ & 0.00 & 0.00 & 0.00 & 0.01 & 0.00 & 0.00 \\
\hline 17 & 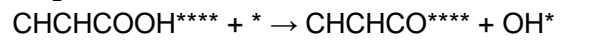 & -0.12 & -0.07 & -0.17 & -0.06 & -0.01 & -0.11 \\
\hline 18 & $\mathrm{CHCHCO}^{\star * \star \star}+3^{*} \rightarrow \mathrm{CHCH}^{\star * \star \star}+\mathrm{CO}^{\star \star *}$ & -0.03 & -0.01 & -0.04 & -0.02 & 0.00 & -0.03 \\
\hline 19 & $\mathrm{CH}_{2} \mathrm{CH}^{\star \star \star}+2^{*} \rightarrow \mathrm{CHCH}^{\star \star \star \star}+\mathrm{H}^{\star}$ & 0.02 & 0.01 & 0.04 & 0.02 & 0.02 & 0.04 \\
\hline 20 & $\mathrm{CH}_{2} \mathrm{CH}_{2}{ }^{\star \star}+2^{\star} \rightarrow \mathrm{CH}_{2} \mathrm{CH}^{\star \star \star}+\mathrm{H}^{\star}$ & 0.01 & -0.01 & 0.01 & 0.01 & 0.01 & 0.01 \\
\hline 21 & $\mathrm{CH}_{2} \mathrm{CH}^{\star \star \star}+* \rightarrow \mathrm{CH}_{2} \mathrm{C}^{\star \star *}+\mathrm{H}^{\star}$ & -0.02 & -0.02 & -0.02 & -0.01 & -0.01 & -0.01 \\
\hline 22 & $\mathrm{CH}_{3} \mathrm{C}^{* * *}+{ }^{*} \rightarrow \mathrm{CH}_{2} \mathrm{C}^{\star * *}+\mathrm{H}^{*}$ & -0.01 & -0.03 & -0.01 & 0.01 & -0.01 & 0.02 \\
\hline 23 & $\mathrm{CH}_{3} \mathrm{CH}^{\star \star \star *}+{ }^{*} \rightarrow \mathrm{CH}_{2} \mathrm{CH}^{\star * *}+\mathrm{H}^{*}$ & -0.03 & -0.03 & -0.06 & 0.00 & -0.02 & -0.03 \\
\hline 24 & $\mathrm{CH}_{3} \mathrm{CH}^{\star \star *}+{ }^{*} \rightarrow \mathrm{CH}_{3} \mathrm{C}^{\star * *}+\mathrm{H}^{*}$ & 0.02 & 0.01 & 0.02 & 0.01 & 0.01 & 0.02 \\
\hline 25 & $\mathrm{CH}_{3} \mathrm{CH}_{2}{ }^{\star \star}+2^{\star} \rightarrow \mathrm{CH}_{3} \mathrm{CH}^{\star \star \star}+\mathrm{H}^{\star}$ & 0.01 & 0.01 & 0.03 & 0.02 & 0.02 & 0.05 \\
\hline 26 & $\mathrm{CH}_{3} \mathrm{CH}_{3}{ }^{*}+2^{*} \rightarrow \mathrm{CH}_{3} \mathrm{CH}_{2}{ }^{* *}+\mathrm{H}^{*}$ & -0.02 & -0.02 & 0.00 & -0.01 & 0.00 & 0.01 \\
\hline 27 & $\mathrm{CH}_{3} \mathrm{CH}_{2}{ }^{* *}+{ }^{*} \rightarrow \mathrm{CH}_{2} \mathrm{CH}_{2}{ }^{* *}+\mathrm{H}^{*}$ & -0.01 & -0.02 & 0.01 & 0.02 & 0.01 & 0.04 \\
\hline 28 & $\mathrm{CH}_{3} \mathrm{CH}_{2} \mathrm{COOH}^{*}+2^{*} \rightarrow \mathrm{CH}_{3} \mathrm{CH}_{2} \mathrm{COO}^{\star *}+\mathrm{H}^{*}$ & -0.07 & -0.03 & -0.09 & -0.03 & -0.01 & -0.06 \\
\hline 29 & $\mathrm{CH}_{3} \mathrm{CH}_{2} \mathrm{COO}^{* *}+{ }^{*} \rightarrow \mathrm{CH}_{3} \mathrm{CH}_{2}{ }^{* *}+\mathrm{CO}_{2}^{*}$ & -0.04 & -0.06 & -0.02 & -0.03 & -0.06 & 0.01 \\
\hline 30 & $\mathrm{CH}_{3} \mathrm{CH} 2 \mathrm{COO}^{\star *}+2^{\star} \rightarrow \mathrm{CH}_{3} \mathrm{CHCOO}^{* * *}+\mathrm{H}^{*}$ & -0.17 & -0.16 & -0.19 & -0.09 & -0.07 & -0.06 \\
\hline 31 & $\mathrm{CH}_{3} \mathrm{CHCOOH}^{\star *}+2^{\star} \rightarrow \mathrm{CH}_{3} \mathrm{CHCOO}^{* * *}+\mathrm{H}^{*}$ & -0.14 & -0.10 & -0.18 & -0.07 & -0.04 & -0.09 \\
\hline 32 & $\mathrm{CH}_{3} \mathrm{CHCOOH}^{\star \star}+3^{\star} \rightarrow \mathrm{CH}_{3} \mathrm{CH}^{\star \star \star}+\mathrm{COOH}^{\star \star}$ & -0.02 & -0.03 & -0.02 & 0.02 & 0.00 & 0.03 \\
\hline 33 & $\mathrm{CH}_{3} \mathrm{CHCOO}^{* \star *}+* \rightarrow \mathrm{CH}_{3} \mathrm{CH}^{\star \star *}+\mathrm{CO}_{2}^{*}$ & 0.12 & 0.08 & 0.17 & 0.10 & 0.06 & 0.13 \\
\hline 34 & $\mathrm{CH}_{3} \mathrm{CHCOO}^{* * *}+{ }^{*} \rightarrow \mathrm{CH}_{3} \mathrm{CCOO}^{* * *}+\mathrm{H}^{*}$ & 0.12 & 0.07 & 0.15 & 0.09 & 0.06 & 0.11 \\
\hline 35 & $\mathrm{CH}_{3} \mathrm{CCOOH}^{* * *}+* \rightarrow \mathrm{CH}_{3} \mathrm{CCOO}^{* * *}+\mathrm{H}^{*}$ & -0.18 & -0.14 & -0.26 & -0.07 & -0.04 & -0.13 \\
\hline 36 & $\mathrm{CH}_{3} \mathrm{CCOOH}^{\star \star \star}+2^{\star} \rightarrow \mathrm{CH}_{3} \mathrm{C}^{\star \star \star}+\mathrm{COOH}^{\star \star}$ & -0.05 & -0.04 & -0.05 & -0.02 & -0.01 & -0.02 \\
\hline 37 & $\mathrm{CH}_{2} \mathrm{CHCOOH}^{\star \star \star *}+* \rightarrow \mathrm{CH}_{2} \mathrm{CH}^{\star \star \star}+\mathrm{COOH}^{\star \star}$ & -0.01 & -0.01 & -0.04 & 0.00 & 0.00 & -0.02 \\
\hline 38 & $\mathrm{CH}_{3} \mathrm{CCOO}^{\star * *}+* \rightarrow \mathrm{CH}_{3} \mathrm{C}^{\star \star *}+\mathrm{CO}_{2}^{*}$ & 0.21 & 0.18 & 0.24 & 0.14 & 0.12 & 0.17 \\
\hline 39 & $\mathrm{COOH}^{\star \star} \rightarrow \mathrm{CO}_{2}^{*}+\mathrm{H}^{\star}$ & -0.18 & -0.13 & -0.25 & -0.14 & -0.09 & -0.20 \\
\hline 40 & $\mathrm{COOH}^{\star \star}+2^{\star} \rightarrow \mathrm{CO}^{\star \star \star}+\mathrm{OH}^{\star}$ & 0.04 & 0.01 & 0.05 & 0.03 & 0.02 & 0.04 \\
\hline 41 & $\mathrm{H}_{2} \mathrm{O}^{*}+{ }^{*} \rightarrow \mathrm{OH}^{*}+\mathrm{H}^{*}$ & -0.01 & 0.02 & -0.05 & -0.01 & 0.01 & -0.03 \\
\hline
\end{tabular}


1

2

3

4

Table S11. Overall, decarbonylation, and decarboxylation turnover frequencies as well as important steady state surface coverages for the HDO of PAC over a Ni(111) catalyst surface model in the gas phase and in liquid water at $473 \mathrm{~K}$. Calculations in water were performed with the help of the iSMS and VASPsol schemes. Also, $a_{i}$ denotes the reaction order with respect to partial pressure/fugacity of component $i$ and $X_{r c}^{n}$ indicates Campbell's degree of rate control for reaction step $n$.

\begin{tabular}{|c|c|c|c|}
\hline Properties & Gas & iSMS & VASPsol \\
\hline DCN TOF & $3.46 \times 10^{-08}$ & $6.70 \times 10^{-09}$ & $1.68 \times 10^{-08}$ \\
\hline DCX TOF & $8.72 \times 10^{-13}$ & $1.47 \times 10^{-11}$ & $1.24 \times 10^{-10}$ \\
\hline Overall TOF $\left(\mathrm{s}^{-1}\right)$ & $3.46 \times 10^{-08}$ & $6.71 \times 10^{-09}$ & $1.69 \times 10^{-08}$ \\
\hline$\theta^{*}$ & 0.011 & 0.003 & 0.008 \\
\hline$\theta_{\mathrm{H}^{*}}$ & 0.631 & 0.206 & 0.418 \\
\hline $\boldsymbol{\theta}_{\mathrm{CO}{ }^{* * *}}$ & 0.357 & 0.789 & 0.474 \\
\hline $\boldsymbol{\theta}_{\mathrm{CH} 3 \mathrm{C}^{*+*}}$ & 0.000 & 0.000 & 0.000 \\
\hline$\theta_{\mathrm{PAC}^{*}}$ & 0.000 & 0.000 & 0.021 \\
\hline$\theta_{\mathrm{CH} 3 \mathrm{CH} 2 \mathrm{COO}^{* *}}$ & 0.001 & 0.000 & 0.010 \\
\hline$a_{c o}$ & 0.0 & -0.7 & -1.0 \\
\hline$a_{H_{2}}$ & -2.2 & -1.4 & -1.2 \\
\hline$a_{P A C}$ & 1.0 & 1.0 & 1.0 \\
\hline$X_{r c}^{1}$ & 0.65 & 0.10 & 0.03 \\
\hline$X_{r c}^{8}$ & 0.26 & 0.01 & 0.68 \\
\hline$X_{r c}^{9}$ & 0.03 & 0.88 & 0.16 \\
\hline
\end{tabular}




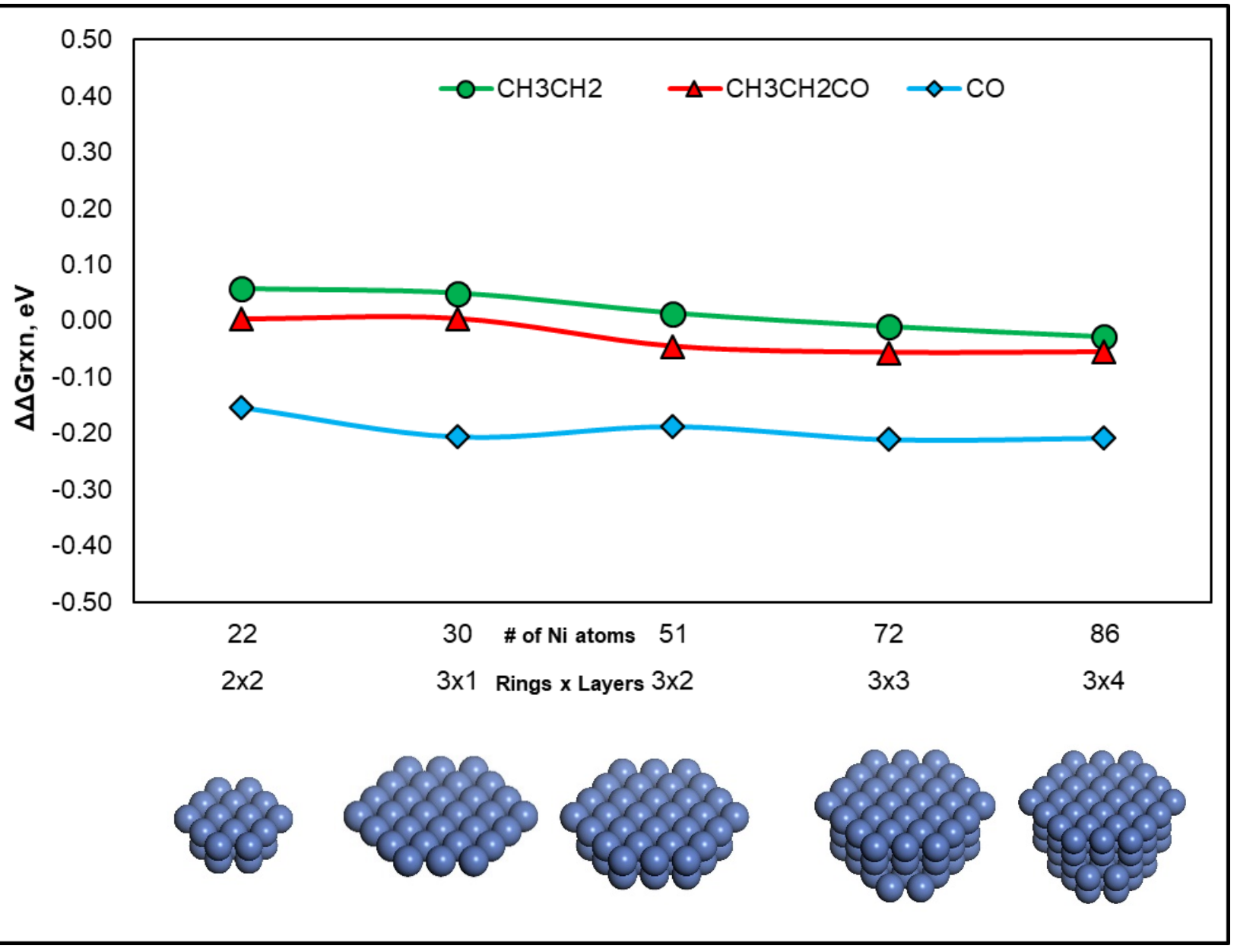

3 Figure S1. Adsorption of different sized molecules $\left(\mathrm{CO}, \mathrm{CH}_{3} \mathrm{CH}_{2}\right.$, and $\left.\mathrm{CH}_{3} \mathrm{CH}_{2} \mathrm{C}\right)$ on a $\mathrm{Ni}(111)$ surface model with 4 various cluster sizes used in iSMS calculations in liquid water at $473 \mathrm{~K}$. We employed a 3x2 cluster model for all 5 surface reactions since the computational cost for larger models increases dramatically while the solvent effect does 6 not change significantly. For adsorption/desorption processes of saturated molecules such as $\mathrm{C}_{2} \mathrm{H}_{4}, \mathrm{C}_{3} \mathrm{H}_{6}, \mathrm{C}_{2} \mathrm{H}_{2}$, $7 \mathrm{CH}_{3} \mathrm{CH}_{2} \mathrm{COOH}, \mathrm{CO}, \mathrm{CO}_{2}, \mathrm{H}, \mathrm{H}_{2} \mathrm{O}$, where error cancellation of solvation effects is smaller than for surface reactions, 8 a $3 \times 4$ model with 86 metal atoms was used. 


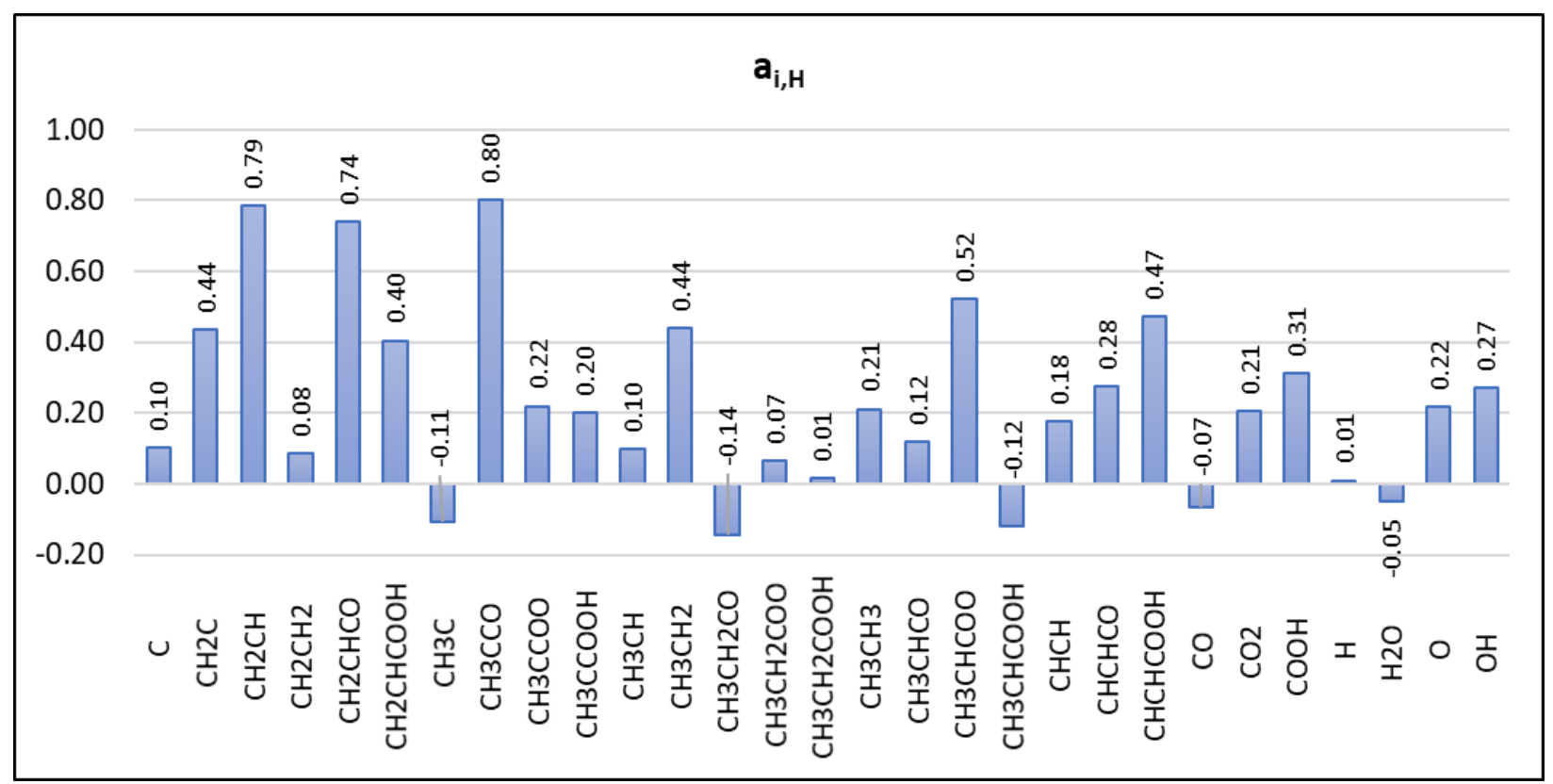

b)

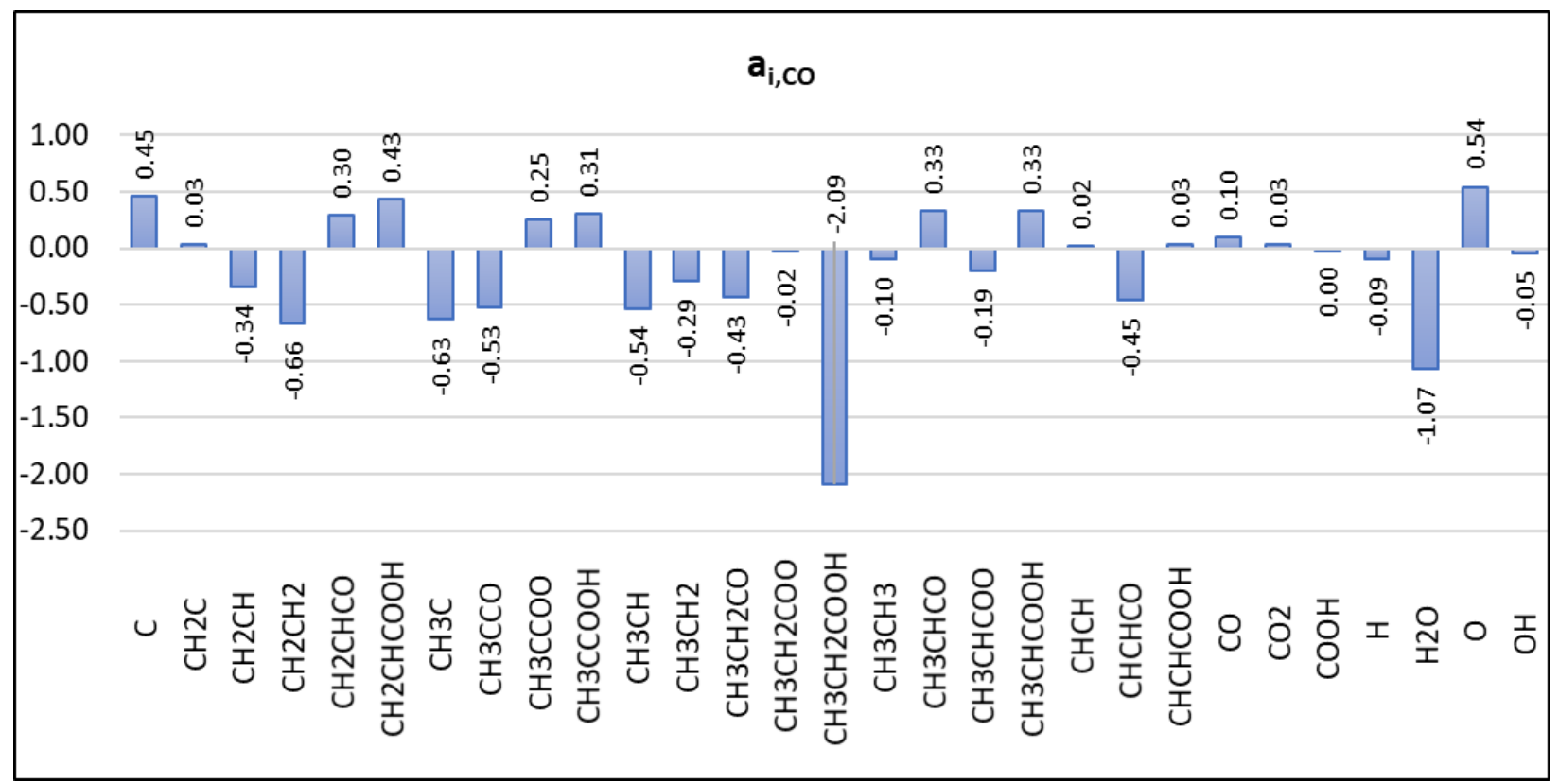

Figure S2. Lateral interaction correction terms for various intermediates using a 1/4 ML coverage of CO and H. Shown are the correction terms of the lateral interaction effect of $\mathrm{H}$ (a) and CO (b) for each individual adsorbed species. 


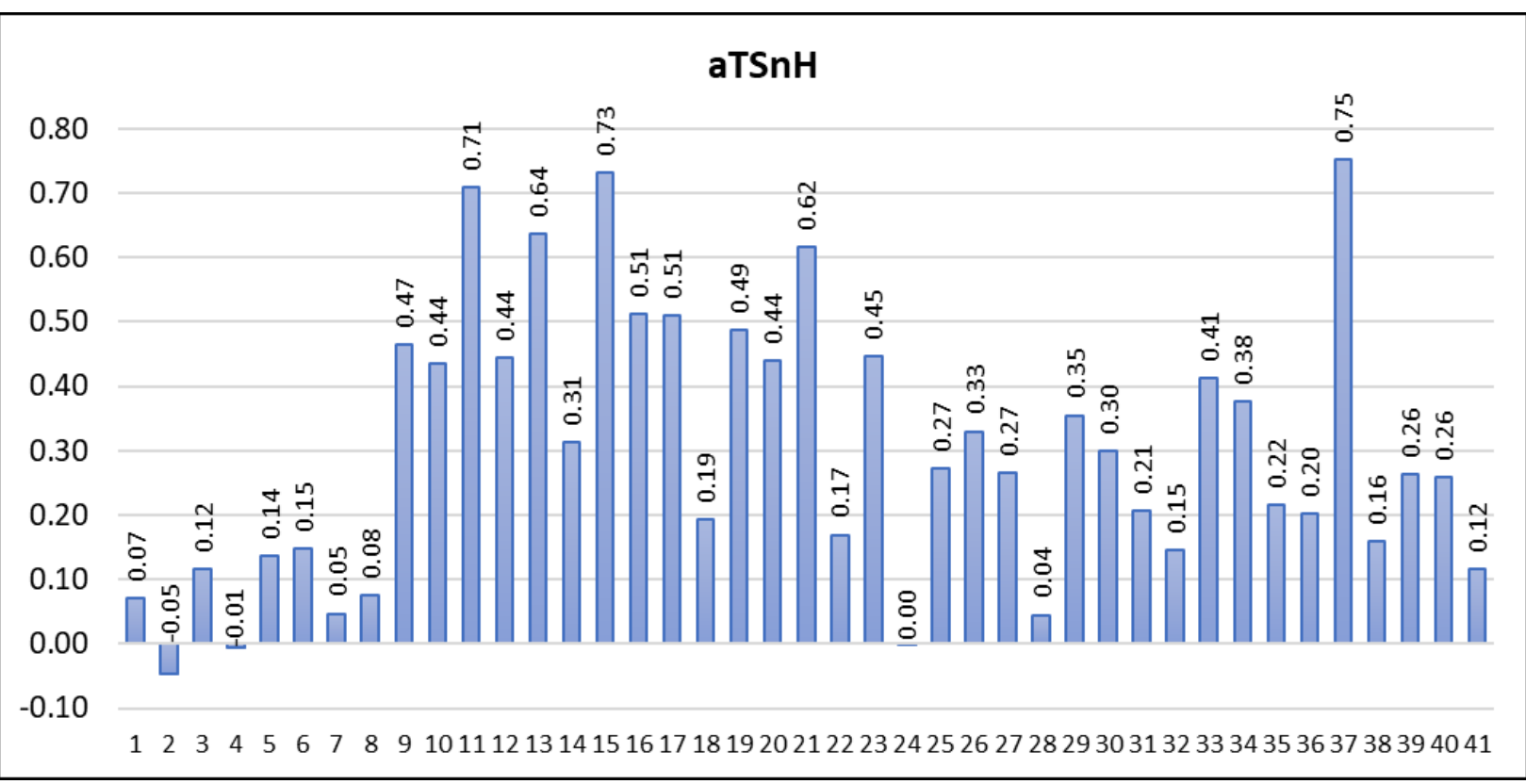

b)

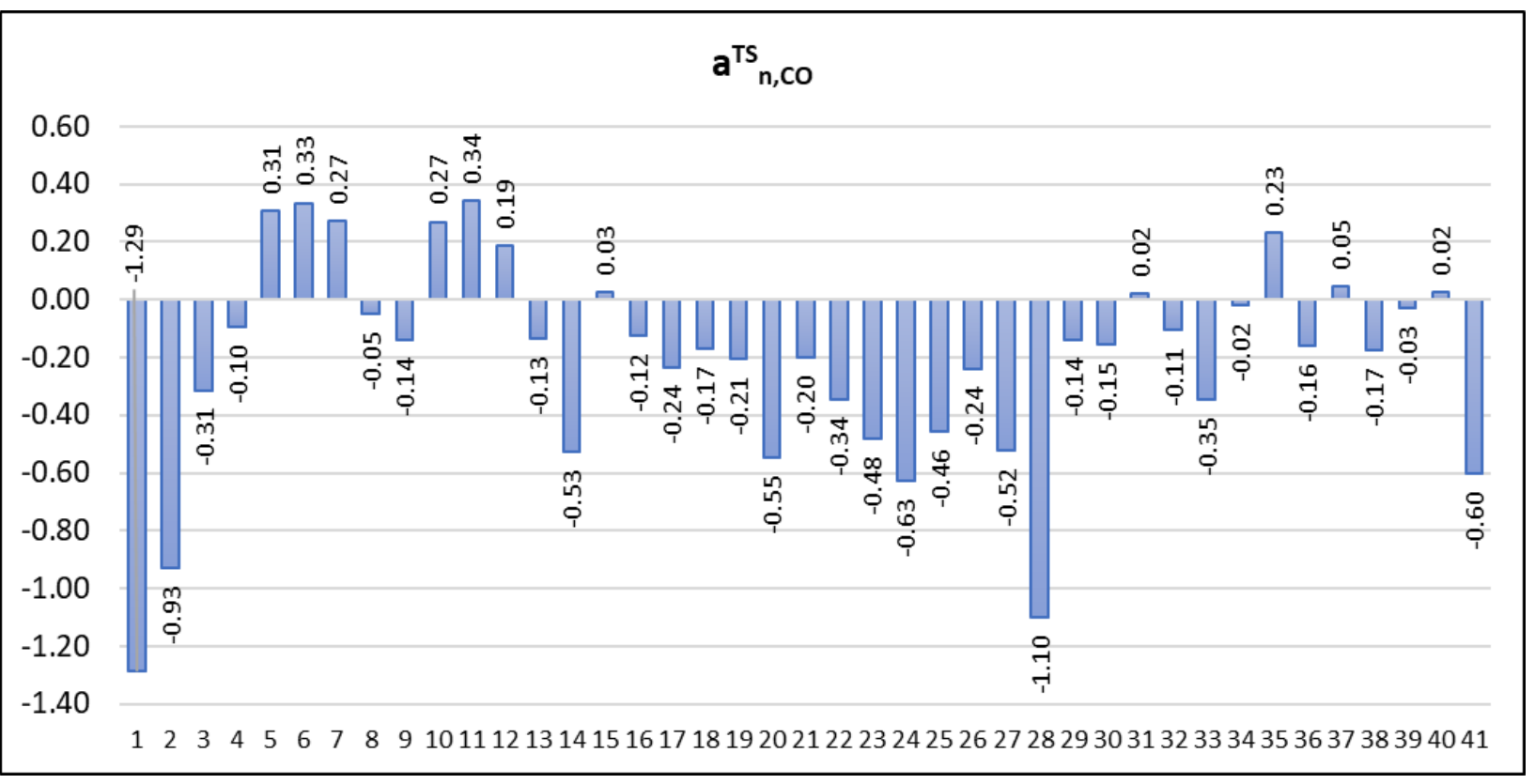

Figure S3. Lateral interaction correction terms for the transition state of each elementary step using 1/4 ML coverages of $\mathrm{CO}$ and $\mathrm{H}$. Shown are the correction terms of the lateral interaction effect of $\mathrm{H}$ (a) and $\mathrm{CO}$ (b) on the transition state of each elementary step. 

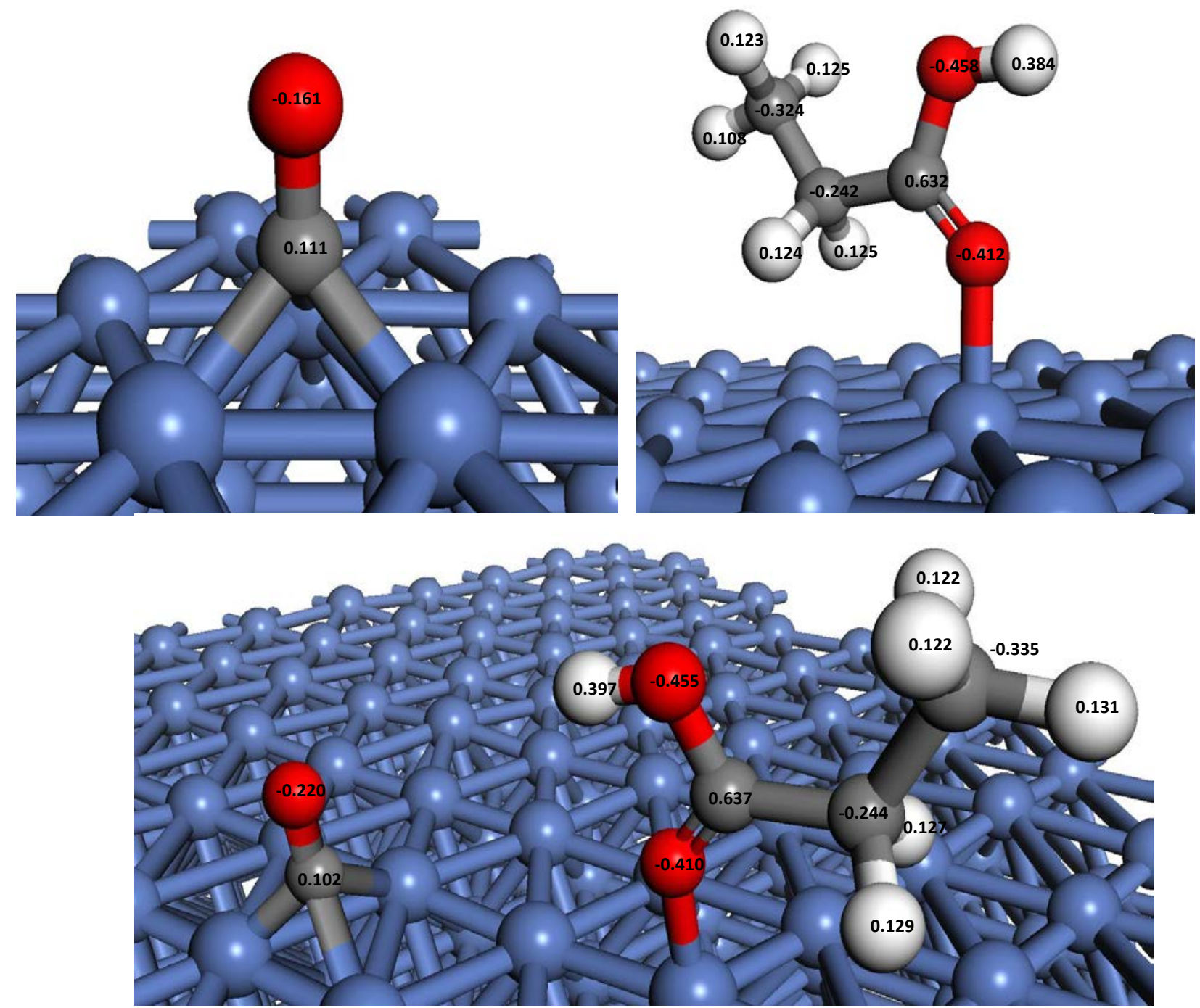

Figure S4. Partial charges computed using the DDEC6 charge model ${ }^{1}$ on adsorbed CO (top left), adsorbed PAC (top right), and coadsorbed CO and PAC on a Ni (111) catalyst surface model. In the coadsorbed state, CO is a Lewis acid, which accepts electrons, while PAC is a Lewis base, which donates electrons. This coadsorbed Lewis acid and base pair explains the attractive interaction between adsorbed CO and PAC. 
a)

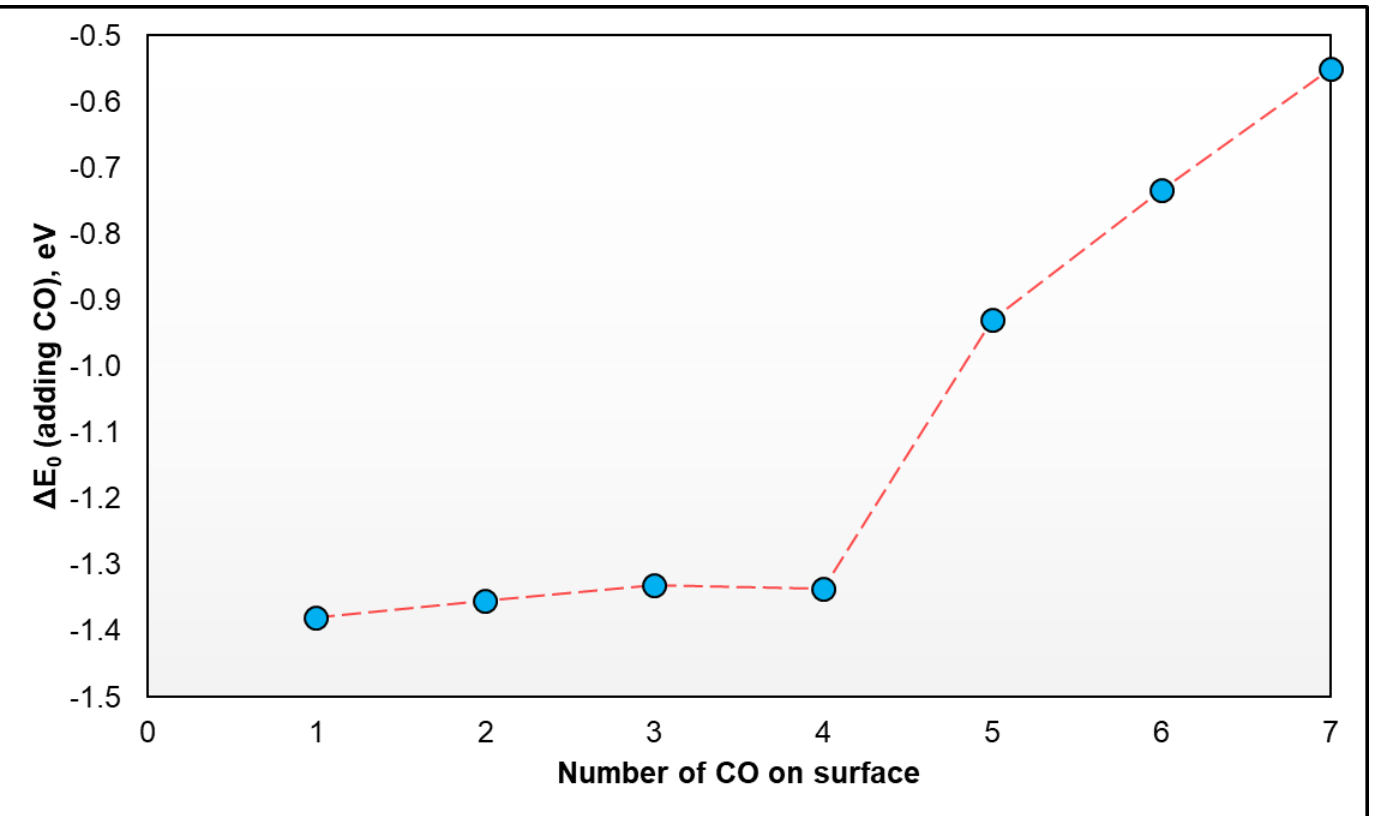

b)

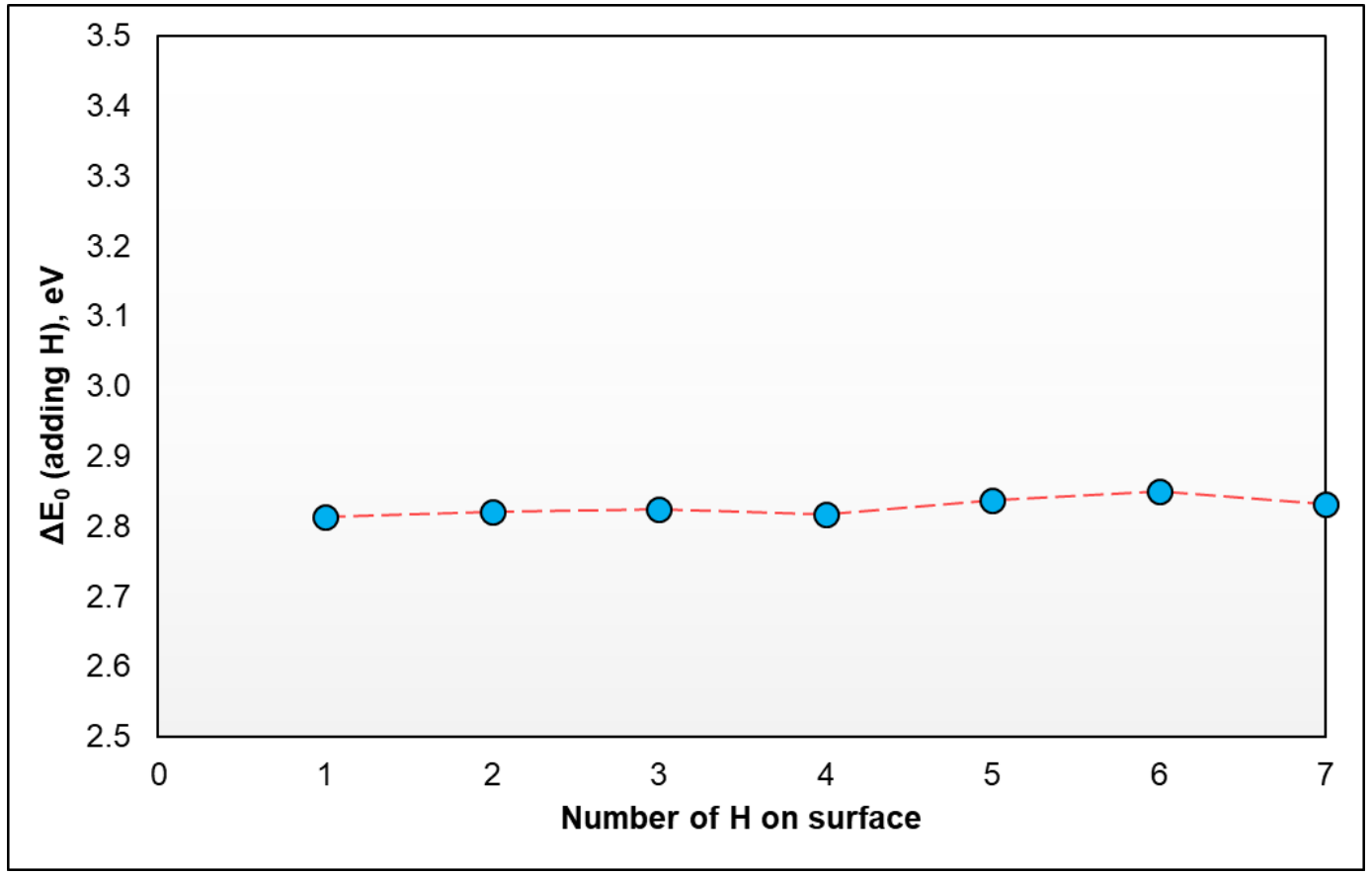

Figure S5. Differential zero-point energy corrected (ZPC) energy of adding a) an extra CO as a function of number of $\mathrm{CO}$ already adsorbed on a $\mathrm{Ni}(111)$ surface containing 12 surface atoms with $(3 \times 2 \sqrt{3})$ periodicity and b) an extra $\mathrm{H}$ as a function of number of $\mathrm{H}$ already adsorbed on the same Ni surface. Adding the fifth $\mathrm{CO}$ increases the energy significantly (by over $0.45 \mathrm{eV}$ ) which we attribute to the fact that the surface is essentially fully covered with $4 \mathrm{CO}$ and adding an extra $\mathrm{CO}$ weakens the bond strength of adsorbed $\mathrm{CO}$ molecules on the surface due to a strong repulsive interaction between them. On the other hand, the differential ZPC barely increases when adding an extra hydrogen atom. Consequently, it can be concluded that a $\mathrm{CO}$ molecule occupies $3 \mathrm{Ni}$ sites on the surface while $\mathrm{H}$ adsorbs on only $1 \mathrm{Ni}$ site. 
1

2 1. T. A. Manz and N. G. Limas, RSC Advances, 2016, 6, 47771-47801. 3 